\title{
Robust Stabilization of a Class of Nonlinear Systems Controlled Over Communication Networks
}

\author{
Gilberto Pin, Gianfranco Fenu, Vittorio Casagrande, Davide Zorzenon, and Thomas Parisini
}

\begin{abstract}
The paper deals with the stabilization of nonlinear systems in which the loop is closed over a lossy nonacknowledged communication network. Given a Regional Inputto-State (ISS) stabilizing state-feedback control law, designed without accounting for the network-induced delays, we propose a non-acknowledged communication policy that allows to deploy the above controller over the network without any modification, while preserving the Regional ISS property. The time-varying delays and packet dropouts occurring on both the up-link and the down-link are compensated through a model-based prediction scheme and a packet-management policy based on time-stamping. The consistency of the prediction, which is a major issue in the context of nonlinear systems with an embedded networked controller, is guaranteed through the exploitation of a novel moveblocking strategy for computing the command sequence to be forwarded to the actuators.
\end{abstract}

\section{INTRODUCTION}

In this paper, we deal with nonlinear dynamic systems in which sensor data and actuator commands are sent through a lossy communication network. Control over communication networks has attracted increasing attention in practical applications, since network technologies provide a convenient way to remotely control distributed plants, with high reconfigurability and simple installation and maintenance. On the other hand, compared to transparent connections, it is well known that the presence of a communication network may affect negatively the performance of the control loop, eventually leading to instability if the design of the control scheme does not explicitly account for the presence of the network itself (see [1], [2], [3], the recent contribution [4] and the monography [5]).

The issue of guaranteeing stability and performance in networked control systems is raised, in particular, by packet dropouts and by the time-varying nature of the transmission

This work has been partially supported by the European Union's Horizon 2020 research and innovation programme under grant agreement No 739551 (KIOS CoE) and by the Italian Ministry for Research in the framework of the 2017 Program for Research Projects of National Interest (PRIN), Grant no. 2017YKXYXJ.

G. Pin is with Dept. of Information Engineering, University of Padova, Italy. (pingilbertedei.unipd.it).

G. Fenu is with the Dept. of Engineering and Architecture at the University of Trieste, Italy (fenu@units.it)

V. Casagrande is with the Dept. of Electronic and Electrical Engineering at University College London, London, UK (vittorio.casagrande.19@ucl.ac.uk).

D. Zorzenon is with the Control Systems Group at Technische Universität Berlin, Germany. (davide.zorzenondtu-berlin.de).

T. Parisini is with the Dept. of Electrical and Electronic Engineering at Imperial College London, UK, with the KIOS Research and Innovation Centre of Excellence, University of Cyprus, and also with the Dept. of Engineering and Architecture at the University of Trieste, Italy (t.parisini@gmail. com) delays, mainly due to the variability of network traffic, to the concurrent access to limited shared resources and to the changing routing paths of the packets sent through the network [6], [7] (see also [8], [9] on the significant issue of concurrency in networked control systems).

Various control strategies have been presented in the literature to design effective networked controllers for linear timeinvariant systems in presence of lossy or delayed communications (see [10], [11], [12], [13], [14]). In particular, many results are focused on characterizing the stability properties of the closed-loop networked system in a probabilistic setting when classical optimal or robust control methodologies are adopted in presence of random transmission delays and packet dropouts [15], [16], [17]. The interested reader is referred to the important paper [18] and to the references cited therein for a deeper insight on the stability analysis of networked control systems. Other interesting papers are [19], [20] and [21] for a deeper insight on the stability analysis of networked control systems in both the stochastic and deterministic frameworks.

Besides guaranteeing the stability of the controller in presence of time-varying delays, another critical issue is the choice of the underlying communication protocol. In this regard, the packet structure of most transmission protocols has important implications from the control point of view, [22]. As far as the reliability of the networked communication is concerned, it is worth pointing out that packet dropouts may occur if a nondeterministic protocol is adopted. Some recent investigation concerning the control over networks with packet losses can be found in [23], [24] and [25]. Assuming that the number of successive dropouts is bounded by a finite upper bound, an effective way to mitigate the effect of packet dropouts consists in using protocols which allow to transmit fewer but more informative packets, containing multiple sensors' data or sequences of control inputs [26], [10], [27], [28], [29]. The control commands can be buffered by the so-called smart actuators, that embed some intelligence to manage packet reordering and synchronization, and to retrieve the control commands from local buffers in case of temporary failure of the communication link. In this context, for example, model predictive controllers represent a natural choice because they are inherently based on the computation of a control input sequence over a future input horizon, that can be easily packeted and forwarded to the actuators (see the seminal paper [30] and the recent contributions [15], [29], [11], [12], [31], [32], [33], [24], [25]). By compensating the network-induced delays through a time-stamping or handshaking policy, it is possible to ensure the recursive feasibility of the optimization problem associated to the model predictive control, which implies, in 
turn, the regional stability of the closed-loop system, [34], [35], [25].

Several sequence-forwarding model-based policies have also been proposed to extend over the network an existing robust stabilizing control law, designed without taking into consideration the presence of the network in the feedback path (see the seminal works [36], [37] and the recent delay-compensation schemes [28], [38], [29], [39]). In these schemes, the major difficulty consists in estimating the current state from delayed partial information with an uncertain model of the process. In the case of varying delays and losses, a further major obstacle is posed by the difficulty to reconstruct the true input sequence applied to the controlled system, since some control commands may be lost in the transmission or be delivered at different times than expected to the actuator nodes. This problem is commonly referred to as prediction consistency issue (see [29], [32], [39]). To guarantee the consistency of the estimates (in other words, that the input sequence used for state reconstruction and prediction matches the one applied to the plant), some sequence-forwarding schemes rely on the assumption that the controller receives back a deterministic notification after each successful packet delivery (acknowledgment), and that perfect synchronization is maintained in the network, permitting to use time-stamps intelligible by all the nodes (see [37], [30], [28], [38] and [34]). These assumptions are hard to verify in general-purpose networks. Moreover, the network synchronization issue is still an open problem, [40], [41]. Therefore, we focus on the case of networks with non-acknowledged communication protocols and with weak synchronization between the plant and the controller.

In this context, we propose a methodology called MoveBlocking Sequence-Forwarding with Plant-Counter Stamping (MBSF-PCS), to extend over a network a conventional robustly-stabilizing controller while preserving its stability properties. In order to guarantee the consistency of the prediction, a sequence-forwarding approach is adopted, together with a move-blocking strategy and a packet-stamping mechanism that relies on the plant's time reference alone. The networked controller driven by the proposed control policy is shown to inherit the Regional Input-to-State Stability (ISS) property of the non-networked pre-designed controller with respect to additive model uncertainties.

The paper is organized as follows: in Section II useful notations, definitions and stability notions are introduced. Then, in Section III we introduce the control objective in formulating some assumptions on the communication network and on the system to be controlled. The devised control policy is presented in Section IV. The stability properties of the resulting networked control systems are then analyzed in Section V. Section VI describes an application of the proposed control strategy. Finally, Section VII reports some concluding remarks and directions of future research efforts.

\section{Notations AND PREliminaries}

This section is aimed at introducing the basic notations used in the paper and the concepts, definitions, and assumptions underlying the characterization of the network nodes.

\section{A. Basic Notations}

Let $\mathbb{R}, \mathbb{R}_{\geq 0}, \mathbb{R}_{>0}, \mathbb{N}$ denote the real, the non-negative real, the strict-positive real,the non-negative integer sets of numbers, respectively. The Euclidean norm is denoted as $|\cdot|$. For any sequence of finite-dimensional vectors $\boldsymbol{v}=$ $\left\{v_{k}\right\}_{k \in \mathbb{N}}$ indexed by a non-negative integer, with $v_{k} \in \mathbb{R}^{n_{v}}$, let us define $\|\boldsymbol{v}\| \triangleq \sup _{k \in \mathbb{N}}\left\{\left|v_{k}\right|\right\}$. The set of discrete-time sequences taking values in some subset $\Upsilon \subset \mathbb{R}^{n_{v}}$ is denoted by $\mathcal{M}_{\Upsilon}$. Given a sequence $\mathbf{u} \in \mathcal{M}_{\Upsilon}$ and two nonnegative integers $k, r \in \mathbb{N}$, with $r \geq k$, we denote as $\mathbf{u}_{k, r}$ the sub-sequence of elements indexed from $k$ to $r$ (i.e., $\left.\mathbf{u}_{k, r} \triangleq\left[u_{k}, \ldots, u_{r}\right] \quad r, k \in \mathbb{N}, r \geq k\right)$.

Definition $2.1\left(\mathcal{K}-, \mathcal{K}_{\infty}, \mathcal{K} \mathcal{L}\right.$-functions): $\quad$ A function $\alpha(\cdot): \mathbb{R}_{\geq 0} \rightarrow \mathbb{R}_{\geq 0}$ belongs to class $\mathcal{K}$ if it is continuous, zero at zero, and strictly increasing. It belongs to class $\mathcal{K}_{\infty}$ if it belongs to class $\mathcal{K}$ and is unbounded.

A function $\beta(\cdot, \cdot): \mathbb{R}_{\geq 0} \times \mathbb{N} \rightarrow \mathbb{R}_{\geq 0}$ belongs to class $\mathcal{K} \mathcal{L}$ if it is non-decreasing in its first argument, non-increasing in its second argument, and $\lim _{s \rightarrow 0} \beta(s, k)=\lim _{k \rightarrow \infty} \beta(s, k)=0$.

Given a $\mathcal{K}_{\infty}$-function $\eta(\cdot)$, the following recursion defines a family of $\mathcal{K}_{\infty}$-functions $\mathcal{G}_{\eta_{i}}(\cdot), i>0$ induced by $\eta(\cdot)$ that will be used throughout the paper:

$$
\left\{\begin{array}{l}
\mathcal{G}_{\eta_{0}}(s) \triangleq 0, \forall s \in \mathbb{R}_{\geq 0} ; \\
\mathcal{G}_{\eta_{i}}(s) \triangleq \eta\left(\mathcal{G}_{\eta_{(i-1)}}(s)\right)+s, \forall s \in \mathbb{R}_{\geq 0}, \quad \forall i \in \mathbb{N} \backslash\{0\}
\end{array}\right.
$$

\section{B. Nodes and Clock Mappings}

In the class of networked control systems dealt with in the paper, a node is understood as a digital computing unit executing repeated operations at discrete-time instants, that is equipped with a local counter (updated at each execution) and that is capable of sending/receiving data to/from other nodes.

With the aim of providing a self consistent characterization of the timing behavior of possibly not synchronized networked elements, let us consider a reference global time (network time) denoted by the continuous variable $t \in \mathbb{R}_{\geq 0}$. The update event of the local counter $k_{z}$ of each $z$-th node of the network is related to the global time by a mapping defined as:

Definition 2.2 (Clock mapping): An injective function

$$
t_{z}(\cdot): \mathbb{N} \rightarrow \mathbb{R}_{\geq 0}
$$

is called a clock mapping if, for some finite $\underline{T}_{z}, \bar{T}_{z} \in$ $\mathbb{R}_{>0}, \underline{T}_{z}<\bar{T}_{z}$, the following inequality is verified:

$$
\underline{T}_{z} \leq t_{z}\left(k_{z}+1\right)-t_{z}\left(k_{z}\right) \leq \bar{T}_{z}, \forall k_{z} \in \mathbb{N} .
$$

Definition 2.3 (Real-time Clock mapping): An injective function

$$
t_{z}(\cdot): \mathbb{N} \rightarrow \mathbb{R}_{\geq 0}
$$

is called a real-time clock mapping if, for some finite $\bar{T}_{z} \in$ $\mathbb{R}_{>0}$, the following identity is verified:

$$
t_{z}\left(k_{z}+1\right)-t_{z}\left(k_{z}\right)=\bar{T}_{z}, \forall k_{z} \in \mathbb{N} .
$$


Let $k_{p}$ denote the counter shared by the plant nodes (sensors, actuators) and let $t_{p}(\cdot)$ be the real-time clock mapping associated to the update of said counter. We denote by $u_{k_{p}} \in \mathbb{R}^{n_{u}} x_{k_{p}} \in \mathbb{R}^{n_{x}}$ respectively the input and the state of the system measured at global time $t_{p}\left(k_{p}\right)$. Since $t_{p}(\cdot)$ is assumed real-time, the plant sampling takes place with constant sampling period $\overline{T_{p}}$. Let the plant's dynamics be described by a discrete-time dynamical system with transition map $F(\cdot, \cdot): \mathbb{R}^{n_{x}} \times \mathbb{R}^{n_{u}} \rightarrow \mathbb{R}^{n_{x}}$, such that:

$$
\left\{\begin{array}{l}
x_{k_{p}+1}=F\left(x_{k_{p}}, u_{k_{p}}\right), \forall k_{p} \in \mathbb{N}, \\
x_{0}=\bar{x}
\end{array}\right.
$$

with $F(0,0)=0$. The following assumptions will be needed.

Assumption 1 (Nominal model, uncertainty bound):

Assume that two compact sets $X \subset \mathbb{R}^{n_{x}}$ and $U \in \mathbb{R}^{n_{u}}$, with $\{0\} \subset X$ and $\{0\} \subset U$, called respectively the modeling domain and the input constraint set, and an approximate state-transition function $\hat{F}(\cdot, \cdot): X \times U \rightarrow X$ are known, such that $\hat{F}(0,0)=0$ and the following bound on the modeling error can be established

$$
|\hat{F}(x, u)-F(x, u)| \leq \bar{d}, \forall(x, u) \in X \times U,
$$

where $\bar{d} \in \mathbb{R}_{\geq 0}$.

Assumption 2 (Uniform continuity w.r.t. x): The approximate discrete state-transition function $\hat{F}(x, u)$ is uniformly continuous with respect to $x$ :

$\left|\hat{F}\left(x^{\prime}, u\right)-\hat{F}\left(x^{\prime \prime}, u\right)\right| \leq \eta_{x}\left(\left|x^{\prime}-x^{\prime \prime}\right|\right), \forall\left(x^{\prime}, x^{\prime \prime}\right) \in X \times X$,

$\forall u \in U$, where $\eta_{x}$ is a class $\mathcal{K}_{\infty}$-function.

Due to (5), there exists a discrete time sequence of additive transition uncertainties $\mathbf{d}_{0, k_{p}}=\left[d_{0}, \ldots, d_{k_{p}}\right]$ such that

$$
\left\{\begin{array}{l}
x_{k_{p}+1}=\hat{F}\left(x_{k_{p}}, u_{k_{p}}\right)+d_{k_{p}}, \forall k_{p} \in \mathbb{N}, \\
x_{0}=\bar{x}
\end{array}\right.
$$

with $\left|d_{k_{p}}\right| \leq \bar{d}, \forall k_{p} \in \mathbb{N}$.

\section{Regional Input-to-State Stability of Discrete-Time Systems}

For the sake of completeness, the notion of Regional Inputto-State Stability for a closed-loop discrete-time system with an exogenous perturbation is recalled. Given a state-feedback map $\kappa(\cdot): \mathbb{R}^{n_{x}} \rightarrow \mathbb{R}^{n_{u}}$, let us consider the discrete-time closed-loop dynamic system

$$
\left\{\begin{array}{l}
x_{k_{p}+1}=\hat{F}\left(x_{k_{p}}, \kappa\left(x_{p}\right)\right)+d_{k_{p}}, \forall k_{p} \in \mathbb{N}, \\
x_{0}=\bar{x} .
\end{array}\right.
$$

The following definition of ISS is provided for discrete-time nonlinear systems of the form (7) .

Definition 2.4 (Regional ISS $\left(X_{0}\right)$ ): Let $X_{0}$ be a subset of $\mathbb{R}^{n_{x}}$, with $\{0\} \subset X_{0}$ and $D \subset \mathbb{R}^{n_{x}}$ compact. If there exist a $\mathcal{K} \mathcal{L}$-function $\beta(\cdot, \cdot)$ and a $\mathcal{K}$-function $\gamma(\cdot)$ such that, for any initial condition $\bar{x} \in X_{0}$, the corresponding state trajectory satisfies

$$
\left.\left|x_{k_{p}}\right| \leq \max \left\{\beta\left(|\bar{x}|, k_{p}\right), \gamma\left(\| \boldsymbol{d}_{0, k_{p}-1}\right) \|\right)\right\},
$$

$\forall k_{p} \in \mathbb{N}$, then the system (7), with $\mathbf{d} \in \mathcal{M}_{D}$, is said to be Regional Input-to-State Stable in $X_{0}\left(\operatorname{ISS}\left(X_{0}\right)\right)$ with respect to bounded additive disturbances.

The Regional-ISS property admits also a characterization in terms of Lyapunov functions (see [42]).

Definition 2.5 (Regional-ISS-Lyapunov Function): Let $X_{0} \subset \mathbb{R}^{n_{x}}, X \subset \mathbb{R}^{n_{x}}$, with $\{0\} \subset X_{0}$ and $X_{0} \subseteq X$ be such that, for any initial condition $\bar{x} \in X_{0}$ and any admissible realization of the disturbance $\forall d \in D$ the corresponding trajectory under (7) is contained in $X$. Then, the function $V(\cdot): X \rightarrow \mathbb{R}_{\geq 0}$ is called a Regional-ISS $\left(X_{0}, X\right)$-Lyapunov function for system (7), if there exist three $\mathcal{K}_{\infty}$-functions $\alpha_{1}(\cdot), \alpha_{2}(\cdot), \alpha_{3}(\cdot)$, and a $\mathcal{K}$-function $\sigma(\cdot)$ such that the following inequalities hold $\forall d \in D$ :

$$
\begin{array}{cc}
V(x) \geq \alpha_{1}(|x|), & \forall x \in X, \\
V(x) \leq \alpha_{2}(|x|), & \forall x \in X, \\
V(\hat{G}(x)+d)-V(x) \leq-\alpha_{3}(|x|)+\sigma(|d|), & \forall x \in X .
\end{array}
$$

Theorem 2.1 (Lyapunov characterization of Regional-ISS): If the system (7) admits a Regional-ISS $\left(X_{0}, X\right)$-Lyapunov function in $X$, then it is Regional-ISS $\left(X_{0}, X\right)$ with respect to $d \in D$.

We assume we know a state-feedback map $\kappa^{*}(\cdot)$, designed for the approximate system's model (6), that verifies the following assumption in the non-networked scenario:

Assumption $3\left(\kappa^{*}\right)$ : There exists a state-feedback map $\kappa^{*}(\cdot): X \rightarrow U$ such that the system (6), in closed loop with $u_{k}=\kappa^{*}\left(x_{k}\right)$, is $\operatorname{ISS}\left(X_{0}\right)$ with respect to $d \in D$.

Assumption 3 implies the existence of a Regional-ISSLyapunov function $V(\cdot): X \rightarrow \mathbb{R}_{\geq 0}$ for the closed-loop system driven by the control law $\kappa^{*}(\cdot)$. Moreover (11) holds with:

$$
V\left(\hat{F}\left(x, \kappa^{*}(x)\right)+d\right)-V(x) \leq-\alpha_{3}(|x|)+\sigma(|d|) .
$$

A supplementary assumption is formulated on the Lyapunov function $V(\cdot)$ of the non-networked closed-loop system.

Assumption 4 (Uniform continuity of $V(\cdot)$ ): There exists a uniformly continuous ISS-Lyapunov function $V(\cdot)$ for the system (6) in closed-loop with $\kappa^{*}(\cdot)$, i.e., there exists a $\mathcal{K}_{\infty^{-}}$ function $\gamma(\cdot)$ such that

$$
\left|V\left(x^{\prime}\right)-V\left(x^{\prime \prime}\right)\right| \leq \gamma\left(\left|x^{\prime}-x^{\prime \prime}\right|\right), \forall\left(x^{\prime}, x^{\prime \prime}\right) \in X \times X .
$$

Note that the Regional-ISS assumption on the nominal closed-loop system allows to address control laws arising from MPC and NMPC that are characterized by local properties related to the feasibility of the constrained optimization they rely on. While addressing the problem of (robust) recursive constraint satisfaction, the present methodology is however clearly not restricted to this kind of controller. Any controller which guarantees the robust invariance of a compact subset of $\mathbb{R}^{n}$ can be dealt with by the proposed networked stabilization scheme. 


\section{Formulation of the Control Problem}

The control objective consists in designing a delaycompensation strategy, relying on the knowledge of a nominally-Regional-ISS-stabilizing controller $k^{*}(\cdot)$ and of the system model, able to preserve the Regional-ISS property in a networked scenario.

The communication between sensors, controller and actuators takes place over a non-acknowledged network with communication delays and bounded data losses. We anticipate that the Lyapunov analysis presented in Section V on the ISS gain-functions will also allow to evaluate the performance loss in the networked scenario and the margin on the tolerable disturbances guaranteeing the satisfaction of the state constraints.

\section{A. General Assumptions on the Network}

With reference to Fig. 1, the network splits the overall system in two different parts: $i$ ) the plant layer and the ii) controller layer. The two layers are connected by two virtual links: the sensor-to-controller link (downlink) and by the controller-to-actuator link (uplink), modeled as nonacknowledged packet-based unidirectional transmission channels affected by random bounded delays and bounded packetloss. The sensor node and the actuator node, belonging to the same physical domain (plant), are assumed to maintain perfect synchronization; this implies that they share the same counter $k_{p}$, with a common mapping $t_{p}(\cdot)$, which is assumed to be real-time with sampling period $\bar{T}_{p}$, according to Definition 2.3. In other words, we assume that the peripherals used to sense the plant and drive the actuators are locally connected to the same computing unit, sharing the same real-time clock.

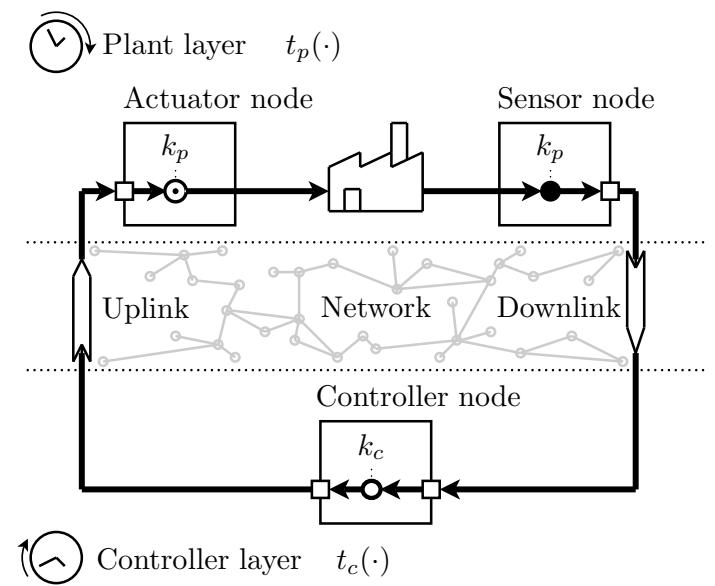

Fig. 1: The networked system dealt with in the paper is partitioned by the network in two physical domains (plant layer and controller layer), with possibly non-synchronized clocks. Each node executes a periodic operation ( $\bullet$ : sensing, O: control, $\odot$ : actuation). The virtual links (uplink and downlink) connecting the two layers are uni-directed and no acknowledgments are used to confirm the arrival of a packet.

On the other hand, the controller is assumed possibly not real-time, meaning that its clock mapping is of the kind specified in Definition 2.2. In particular, we will consider the cycle-time bounded from above by known finite constant $\bar{T}_{c}$.
While the local counters $k_{p}$ and $k_{c}$ are known to the computing nodes, the functions $t_{p}(\cdot)$ and $t_{c}(\cdot)$ mapping the counter-updates to the global time are not known to the NCS's components. The clock mappings, indeed, are not used by the proposed policy, but are instrumental to analyze the networked asynchronous behavior of the NCS components.

\section{B. Plant-Counter Stamping}

The time-stamping-based management of incoming data (hierarchical organization, update and discard of incoming information based on time-stamps applied by the sender) represents a key ingredient of many architectures proposed in the literature for ensuring the stability of a networked control system in absence of acknowledgments. The benefits of using time-stamping in NCS's are well documented (see [1], [43], [34], [35] and the references therein); however the timestamping requires, in general, that all the nodes of the network have access to a common system's clock, or that a proper clock synchronization service is provided by the underlying network protocol. This task can be achieved in different ways (see, e.g., [44], [45]), however, unless a dedicated physical link for synchronization is established between two nodes, the exact synchronization is difficult to obtain.

In order to overcome the clock-synchronization issue, an incoming data management strategy named Plant-Counter Stamping (PCS) is proposed in this work, which is based on the fact that the sensor node applies to the data sent to controller a stamp corresponding to the local counter $k_{p}$. Then, the controller stamps the control data for the actuators with the same plant-counter stamp $k_{p}$. In this way, the actuator receives information about the sampling instant of the source sensory data used to compute the control command.

Remark 3.1: It is worth remarking that the above policy is not properly a time-stamping one, since the mapping to the global time $t_{p}(\cdot)$ is not needed for the stamping; moreover, the controller does not stamp the data for the actuators with the local information. In practice, the stamp applied to sensory data carries information on the plant counter value corresponding to the plant sampling instant. The controller simply forwards this information, which relates to the sensor data, not the time of control computation. In this setting, each node stores the received data in a local memory, until a packet with a more recent plant-counter-stamp is received, replacing the old data.

\section{Bounds on Network Delays, Packet Losses, and Implica- tions}

We assume that the number of consecutive dropouts is bounded by a finite integer $\bar{L} \in \mathbb{N}$ in both the uplink and the downlink. Not being acknowledged upon the successful reception of the packet by the recipient, each node at any cycle sends a data packet to the recipient even if said data remained unchanged from the previous round. Due to packet dropouts and delays it may happen that the actuator node does not receive updated commands from the controller for a number of cycles. In the following, we will determine an upper bound on this blanking time, in terms of actuator cycles. 
Let us first analyze the case of the downlink. We denote by $\mathbb{K}_{p} \subseteq \mathbb{N}$ the set of all cycles of the sensor node whose packets reach the controller. It means that a message sent at a cycle $k_{p} \in\left(\mathbb{K}_{p}\right)$ is dropped by the network and does not reach the recipient. Moreover, let $\tau_{p}\left(k_{p}\right), k_{p} \in \mathbb{K}_{p}$ denote the time taken by the packet sent at cycle $k_{p}$ to reach the controller. We assume that $\tau_{p}\left(k_{p}\right)$ satisfies the bound:

$$
\tau_{p}\left(k_{p}\right) \leq \bar{\tau}_{p}, \forall k_{p} \in \mathbb{K}_{p},
$$

for some finite $\bar{\tau}_{p} \in \mathbb{R}_{\geq 0}$. Consider now the case that the sensor node at the $k_{p}^{\prime}$-th cycle, with $k_{p}^{\prime} \in \mathbb{K}_{p}$, sends a packet to the controller and let $k_{c}^{\prime}$ be the controller cycle at which the control computation has been completed by exploiting said sensory packet. Then, letting the controller computation-time be upper bounded by $\bar{T}_{c}$, the time at which the computation of the control action is completed, $t_{c}\left(k_{c}^{\prime}\right)$, can be bounded by $t_{p}\left(k_{p}^{\prime}\right) \leq t_{c}\left(k_{c}^{\prime}\right) \leq t_{p}\left(k_{p}^{\prime}\right)+\tau_{p}\left(k_{p}^{\prime}\right)+\bar{T}_{c} \leq t_{p}\left(k_{p}^{\prime}\right)+\bar{\tau}_{p}+\bar{T}_{c}$.

The lower bound corresponds to the ideal situation of zero communication delay in the downlink. We assume that at cycle $k_{c}^{\prime}$, as soon as the control computation has ended, the controller sends the control action to the actuator node. In the ideal case of zero-delays in the uplink and downlink, no dropouts and zero execution time of the controller cycle, the actuator may receive the control command at earliest time

$$
t_{p}\left(k_{p}^{*}\right) \geq t_{p}\left(k_{p}^{\prime}\right)
$$

where $k_{p}^{*}$ represents the cycle of reception at the actuator node.

After the successful delivery of a sensory packet sampled at cycle $k_{p}^{\prime} \in \mathbb{K}_{p}$, at most $\bar{L}$ consecutive dropouts may occur before a new packet, collected by the sensor node at time $k_{p}^{\prime \prime} \in \mathbb{K}_{p}$ is successfully delivered, that is $\forall k_{p}^{\prime} \in \mathbb{K}_{p}, \exists k_{p}^{\prime \prime} \in \mathbb{K}_{p}: k_{p}^{\prime \prime}>k_{p}^{\prime},\left|k_{p}^{\prime}-k_{p}^{\prime \prime}\right| \leq \bar{L}+1$. Therefore, the next sensor cycle after $k_{p}^{\prime}$ with successful delivery to the controller satisfies the bound:

$$
k_{p}^{\prime \prime} \leq k_{p}^{\prime}+(\bar{L}+1) .
$$

In view of the bound (3) on the clock mapping $t_{p}(\cdot)$, it follows that

$$
t_{p}\left(k_{p}^{\prime \prime}\right) \leq t_{p}\left(k_{p}^{\prime}\right)+(\bar{L}+1) \bar{T}_{p},
$$

for some finite $\bar{T}_{p}$. Let $k_{c}^{\prime \prime}$ be the controller cycle at which a new control computation is completed, using the plant sensed at the sensor cycle $k_{p}^{\prime \prime}$. Then:

$$
\begin{aligned}
t_{c}\left(k_{c}^{\prime \prime}\right) & \leq t_{p}\left(k_{p}^{\prime \prime}\right)+\bar{\tau}_{p}+\bar{T}_{c} . \\
& \leq t_{p}\left(k_{p}^{\prime}\right)+\bar{\tau}_{p}+\bar{T}_{c}+(\bar{L}+1) \bar{T}_{p} .
\end{aligned}
$$

At cycle $k_{c}^{\prime \prime}$, as soon as the control computation has ended, the controller tries to send the control action to the actuator node, stamping it with $k_{p}^{\prime \prime}$, i.e., the plant-counter of the source sensory data. The data transmission delay in the uplink satisfies the bound:

$$
\tau_{c}\left(k_{c}\right) \leq \bar{\tau}_{c}, \forall k_{c} \in \mathbb{K}_{c}
$$

for some finite $\bar{\tau}_{c} \in \mathbb{R}_{\geq 0}$, where $\mathbb{K}_{c} \subseteq \mathbb{N}$ denotes the set of controller cycles whose packets are successfully delivered to the actuator. In the worst-case scenario, however, the controller may not receive new sensory data for the next $\operatorname{ceil}\left(\bar{L} \bar{T}_{p} / \underline{T}_{c}\right)$ cycles (due to losses in the downlink) and it may take up to $\bar{L}+1$ cycles to successfully deliver the packet, due to losses in the uplink. Therefore, letting

$$
\bar{L}_{c} \triangleq \max \left(\bar{L}, \operatorname{ceil}\left(\bar{L} \bar{T}_{p} / \underline{T}_{c}\right)\right)
$$

and denoting by $k_{p}^{* *}$ the actuator's cycle at which the control packet is received, we get the bound:

$$
\begin{aligned}
t_{p}\left(k_{p}^{* *}\right) & \leq t_{c}\left(k_{c}^{\prime \prime}\right)+\left(\bar{L}_{c}+1\right) \bar{T}_{c} \\
& \leq t_{p}\left(k_{p}^{\prime}\right)+\bar{\tau}_{p}+\bar{T}_{c}+(\bar{L}+1) \bar{T}_{p}+\bar{\tau}_{c}+\left(\bar{L}_{c}+1\right) \bar{T}_{c} .
\end{aligned}
$$

Note that, by introducing the constant $\bar{\tau}_{r t}$ defined as:

$$
\bar{\tau}_{r t} \triangleq \bar{\tau}_{p}+\bar{\tau}_{c}+\bar{T}_{c}+(\bar{L}+1) T_{p}+\left(\bar{L}_{c}+1\right) \bar{T}_{c},
$$

the time-lapse between $t_{p}\left(k_{p}^{*}\right)$ and $t_{p}\left(k_{p}^{* *}\right)$, that is the timeframe between two successive updates of the actuator, in view of (14) and (18) can be upper bounded by $\bar{\tau}_{r t}$ as follows:

$$
t_{p}\left(k_{p}^{* *}\right)-t_{p}\left(k_{p}^{*}\right) \leq \bar{\tau}_{r t} .
$$

The bound $\bar{\tau}_{r t}$ is also known as maximum round-trip-time, since it represents the maximum time taken by a packet sent from the plant layer (sensor-node) to return to same layer (actuator-node). Note that between cycles $k_{p}^{*}$ and $k_{p}^{* *}$ the actuator node does not receive any update. The harmful presence of such a blanking time can be mitigated by a sequence-forwarding policy (see [36], [37], [28], [38], [29] and [39]). That is, the controller sends more informative packets containing a sequence of $N_{p} \in \mathbb{N}$ control moves instead of a single control command, with

$$
N_{p} \geq \operatorname{ceil}\left(\frac{\bar{\tau}_{r t}}{\bar{T}_{p}}\right),
$$

to avoid the buffer overrun issue.

\section{The Move-Blocking SEQUence-Forwarding POLICY}

According to the previous analysis on network delays, the proposed control strategy requires the controller node to compute, at any $k_{c}^{\prime \prime}$-th cycle, a sequence of $N_{p}+1$ control inputs:

$$
\mathbf{u}^{\hat{F}}\left(k_{c}^{\prime \prime}\right)_{k_{p}^{\prime \prime}, k_{p}^{\prime \prime}+N_{p}}=\left[u^{\hat{F}}\left(k_{c}^{\prime \prime}\right)_{k_{p}^{\prime \prime}}, \ldots, u^{\hat{F}}\left(k_{c}^{\prime \prime}\right)_{k_{p}^{\prime \prime}+N_{p}}\right],
$$

by exploiting the sensor data collected at time $k_{p}^{\prime \prime}$. The superscript $\hat{F}$ denotes the fact that this sequence is computed by the controller thanks to a model-based prediction of the system's trajectory. The index enclosed by round brackets (e.g. $k_{c}^{\prime \prime}$ in this case) denotes the controller cycle at which the sequence is computed, while the subscripted indices on the elements on the right denote the plant's cycles at which the relative control move should be applied. The elements of the sequence with subscript larger than $k_{p}^{\prime \prime}$ refer to control moves that should be applied forward-in-time by the actuator node, if needed, to recover the blanking time. While modelbased packet forwarding is a well established technique for delay compensation, the originality of the present contribution 
resides in the way the computation of forward control moves is carried out, that enables the present strategy to be applied also to controllers involving constrained optimization, for which recursive feasibility must be guaranteed.

In the conventional discrete-time state-feedback control setting (with transparent communication), the control move to be applied at the $k_{p}^{* *}$-th cycle of the actuating node relies on the current state value: $u\left(k_{p}^{* *}\right)=\kappa^{*}\left(x_{k_{p}^{* *}}\right)$. Conversely, in the networked setting, the input applied at time $k_{p}^{* *}$ may have relied at a time $k_{c}^{\prime \prime}$, then $u_{k_{p}^{* *}}$ should be computed based on the available state measurement $x_{k_{p}^{\prime \prime}}$ thanks to a model-based state prediction $\hat{x}_{k_{p}^{* *}}$. Nonetheless, to estimate a future state $\hat{x}_{k_{p}^{* *}}$ from the available measurement $x_{k_{p}^{\prime \prime}}$, it is important to exactly know the input sequence $\mathbf{u}_{k_{p}^{\prime \prime}, k_{p}^{* *}-1}$ that will be driving the system (6) from $k_{p}^{\prime \prime}$ to $k_{p}^{* *}-1$.

As far as the state reconstruction/estimation is concerned, the uncertainty on the future input sequence may represent a severe issue, especially when the plant response is very sensitive to the control input, or when significant variations occur between consecutive control actions, such in the case of discontinuous control laws. To force determinism on the control moves that will be applied (a.k.a. "prediction consistency"), we resort to a move-blocking policy, formally described in the following.

Given the sequence computed at the $k_{c}^{\prime}$-th cycle by the controller (exploiting sensory data stamped with $k_{p}^{\prime}$ ):

$$
\mathbf{u}^{\hat{F}}\left(k_{c}^{\prime}\right)_{k_{p}^{\prime}, k_{p}^{\prime}+N_{p}}=\left[u^{\hat{F}}\left(k_{c}^{\prime}\right)_{k_{p}^{\prime}}, \cdots, u^{\hat{F}}\left(k_{c}^{\prime}\right)_{k_{p}^{\prime}+N_{p}}\right],
$$

let the new sequence to be computed at cycle $k_{c}^{\prime \prime}$ with sensory data collected at plant's cycle $k_{p}^{\prime \prime}$, be written for convenience as the collation of two subsequences:

$$
\begin{aligned}
& \mathbf{u}^{\hat{F}}\left(k_{c}^{\prime \prime}\right)_{k_{p}^{\prime \prime}, k_{p}^{\prime \prime}+N_{p}}=\left[\mathbf{u}^{\hat{F}}\left(k_{c}^{\prime \prime}\right)_{k_{p}^{\prime \prime}, k_{p}^{\prime}+N_{p}},\right. \\
& \left.\mathbf{u}^{\hat{F}}\left(k_{c}^{\prime \prime}\right)_{k_{p}^{\prime}+N_{p}+1, k_{p}^{\prime \prime}+N_{p}}\right] .
\end{aligned}
$$

The first subsequence is obtained by reusing the last elements of the previous control sequence (21):

$$
\mathbf{u}^{\hat{F}}\left(k_{c}^{\prime \prime}\right)_{k_{p}^{\prime \prime}, k_{p}^{\prime}+N_{p}}=\left[u^{\hat{F}}\left(k_{c}^{\prime}\right)_{k_{p}^{\prime \prime}}, \ldots, u^{\hat{F}}\left(k_{c}^{\prime}\right)_{k_{p}^{\prime}+N_{p}}\right] .
$$

By using $\mathbf{u}^{\hat{F}}\left(k_{c}^{\prime \prime}\right)_{k_{p}^{\prime \prime}, k_{p}^{\prime}+N_{p}}$, the state estimate $\hat{x}_{k_{p}^{\prime}+N_{p}+1}$ can be obtained by the recursion:

$$
\begin{aligned}
& \hat{x}_{k_{p}^{\prime \prime}}=x_{k_{p}^{\prime \prime}}, \\
& \left\{\begin{array}{c}
\hat{x}_{k_{p}^{\prime \prime}+i}=\hat{F}\left(\hat{x}_{k_{p}^{\prime \prime}+i-1}, u^{\hat{F}}\left(k_{c}^{\prime \prime}\right)_{k_{p}^{\prime \prime}+i-1}\right), \\
\forall i \in\left\{1, \ldots, k_{p}^{\prime}+N_{p}+1-k_{p}^{\prime \prime}\right\} .
\end{array}\right.
\end{aligned}
$$

Then, starting with $\hat{x}_{k_{p}^{\prime}+N_{p}+1}$, the second subsequence is computed by the following model-based iteration that exploits the control law $\kappa^{*}(\cdot)$ :

$$
\left\{\begin{array}{l}
u^{\hat{F}}\left(k_{c}^{\prime \prime}\right)_{k_{p}^{\prime}+N_{p}+j}=\kappa^{*}\left(\hat{x}_{k_{p}^{\prime}+N_{p}+j}\right), \\
\hat{x}_{k_{p}^{\prime}+N_{p}+1+j}=\hat{F}\left(\hat{x}_{k_{p}^{\prime}+N_{p}+j}, u^{\hat{F}}\left(k_{c}^{\prime \prime}\right)_{k_{p}^{\prime}+N_{p}+j}\right), \\
\quad \forall j \in\left\{1, \ldots, k_{p}^{\prime \prime}-k_{p}^{\prime}\right\} .
\end{array}\right.
$$

The move-blocking policy adopted for the input sequence computation (22), implies that the control sequence that will be applied to the plant is fully determined and known by the controller. Thus, the prediction consistency of the proposed scheme is guaranteed by construction.

In the next section, the stability properties of the devised MBSF methodology with PCS delay compensation are addressed.

\section{InPUT-To-State Stability PReservation Under THE MBSF-PCS POLICY}

To carry out the stability analysis, let us adopt an actuatorcentric point of view. Let $x_{k_{p}^{* *}}$ be the true system's state in correspondence of the $k_{p}^{* *}$-th cycle of the actuator task and let $\hat{x}_{k_{p}^{* *}}$ be a the state prediction done by the controller. In view of this bound on the round-trip time, a worst-case analysis on the state estimation error can be carried out.

Lemma 5.1 (Estimation error bound): Under Assumptions 1 and 2, for any actuator cycle $k_{p}^{* *} \in \mathbb{N}$, the state estimate $\hat{x}_{k_{p}^{* *}}$ initialized by $x\left(k_{p}^{\prime \prime}\right)$, with $k_{p}^{* *}-\bar{\tau}_{r t} \leq k_{p}^{\prime \prime} \leq k_{p}^{* *}$, and computed through model-based recursions (23) and (24), is affected by an error that satisfies the norm bound

$$
\left|\hat{x}_{k_{p}^{* *}}-x_{k_{p}^{* *}}\right| \leq \Gamma_{\eta_{\bar{\tau}_{r t}}}(\bar{d}),
$$

where

$$
\Gamma_{\eta_{j}}(s) \triangleq \max _{i \in\{0, \ldots, j\}}\left\{\mathcal{G}_{\eta_{i}}(s)\right\}, \forall s \in \mathbb{R}_{\geq 0},
$$

is a $\mathcal{K}_{\infty}$-function, with $\mathcal{G}_{\eta_{i}}(\cdot), i \in\{0, \ldots, j\}$ defined in (1).

Proof: Given the state measurement $x_{k_{p}^{\prime \prime}}$, the control sequence $\mathbf{u}_{k_{p}^{\prime \prime}, k_{p}^{* *}}$, and the uncertainty realization $\mathbf{d}_{k_{p}^{\prime \prime}, k_{p}^{* *}}$, in view of Assumption 2 and (5), the state estimation error $\hat{x}_{k_{p}^{\prime \prime}+1}-x_{k_{p}^{\prime \prime}+1}$ can be bounded in norm by

$$
\begin{aligned}
& \left|\hat{x}_{k_{p}^{\prime \prime}+1}-x_{k_{p}^{\prime \prime}+1}\right|= \\
& \quad=\left|\hat{F}\left(x_{k_{p}^{\prime \prime}}, u_{k_{p}^{\prime \prime}}^{\hat{F}}\right)-F_{p}\left(x_{k_{p}^{\prime \prime}}, u_{k_{p}^{\prime \prime}}^{\hat{T}}\right)\right| \\
& \quad \leq\left|d_{k_{p}^{\prime \prime}}\right| \leq \bar{d}=\mathcal{G}_{\eta_{1}}(\bar{d}) .
\end{aligned}
$$

At the next step, by the triangle inequality, the following equality holds:

$$
\begin{aligned}
& \left|\hat{x}_{k_{p}^{\prime \prime}+2}-x_{k_{p}^{\prime \prime}+2}\right|= \\
& \quad=\left|\hat{F}\left(\hat{x}_{k_{p}^{\prime \prime}+1}, u^{\hat{F}}\left(k_{c}^{\prime \prime}\right)_{k_{p}^{\prime \prime}+1}\right)-F_{p}\left(x_{k_{p}^{\prime \prime}+1}, u_{k_{p}^{\prime \prime}+1}\right)\right| .
\end{aligned}
$$

Since the move-blocking policy yields $u_{k_{p}^{\prime \prime}+1}=u^{\hat{F}}\left(k_{c}^{\prime \prime}\right)_{k_{p}^{\prime \prime}+1}$ (the truly applied control sequence coincides with the one known to the controller), then

$$
\begin{aligned}
& \left|\hat{x}_{k_{p}^{\prime \prime}+2}-x_{k_{p}^{\prime \prime}+2}\right| \leq \\
& \quad \leq\left|\hat{F}\left(\hat{x}_{k_{p}^{\prime \prime}+1}, u_{k_{p}^{\prime \prime}+1}\right)-\hat{F}\left(x_{k_{p}^{\prime \prime}+1}, u_{k_{p}^{\prime \prime}+1}\right)\right| \\
& \quad+\left|\hat{F}\left(x_{k_{p}^{\prime \prime}+1}, u_{k_{p}^{\prime \prime}+1}\right)-F_{p}\left(x_{k_{p}^{\prime \prime}+1}, u_{k_{p}^{\prime \prime}+1}\right)\right| \\
& \quad \leq \eta\left(\left|\hat{x}_{k_{p}^{\prime \prime}+1}-x_{k_{p}^{\prime \prime}+1}\right|\right)+\left|d_{k_{p}^{\prime \prime}+1}\right| \\
& \quad \leq \eta(\bar{d})+\bar{d}=\mathcal{G}_{\eta_{2}}(\bar{d}) .
\end{aligned}
$$

By applying recursively the triangle inequality, it follows that

$$
\begin{aligned}
& \left|\hat{x}_{k_{p}^{\prime \prime}+i}-x_{k_{p}^{\prime \prime}+i}\right|= \\
& \quad \leq \eta\left(\left|\hat{x}_{k_{p}^{\prime \prime}+i-1}-x_{k_{p}^{\prime \prime}+i-1}\right|\right)+\left|d_{k_{p}^{\prime \prime}+i-1}\right| \\
& \quad \leq \eta\left(\mathcal{G}_{\eta_{(i-1)}}(\bar{d})\right)+\bar{d}=\mathcal{G}_{\eta_{i}}(\bar{d}), \quad \forall i \in\left\{1, \ldots, N_{p}+1\right\} .
\end{aligned}
$$


Being $k_{p}^{* *}-k_{p}^{\prime \prime} \leq \bar{\tau}_{r t}$, the worst-case bound can be evaluated as:

$$
\left|\hat{x}_{k_{p}^{* *}}-x_{k_{p}^{* *}}\right| \leq \max _{i \in\left\{0, \ldots, \bar{\tau}_{r t}\right\}}\left\{\mathcal{G}_{\eta_{i}}(\bar{d})\right\}, \forall k_{p}^{* *} \in \mathbb{N},
$$

which can be written in compact form as in the statement of the lemma.

Theorem 5.1 (Regional ISS $\left(\Xi_{0}\right)$ ): Suppose that Assumptions $1 \div 4$ hold. Then, there exists a non-empty set of initial conditions $\Xi_{0} \subseteq X_{0}$ such that the system controlled by the proposed MBSF policy is $\operatorname{ISS}\left(\Xi_{0}\right)$ with respect to bounded additive uncertainties affecting the state transition function.

Proof: The proof consists in showing that the $\mathrm{Re}$ gional $\operatorname{ISS}\left(X_{0}, X\right)$-Lyapunov function $V(\cdot)$ of the system (6) in closed-loop with the control law $\kappa^{*}(\cdot)$ is a Regional$\operatorname{ISS}\left(\Xi_{0}, X\right)$-Lyapunov function also for the system in closedloop with the MBSF policy in the networked scenario, with $\Xi_{0} \subseteq X_{0}$. That is, the ISS property is inherited by the networked system with a contraction of the allowable set of initial conditions. Being (9) and (10) trivial to prove, it is sufficient to show that the closed-loop trajectories are indefinitely contained in $X$ and that the 3rd ISS-Lyapunov inequality, (11), also holds for the networked closed-loop system, that is

$$
\begin{aligned}
V\left(F\left(x_{k_{p}^{* *}}, u_{k_{p}^{* *}}\right)\right) & -V\left(x_{k_{p}^{* *}}\right) \\
= & V\left(F\left(x_{k_{p}^{* *}}, \kappa^{*}\left(\hat{x}_{k_{p}^{* *}}\right)\right)\right)-V\left(x_{k_{p}^{* *}}\right) \\
\leq & -\hat{\alpha}_{3}\left(\left|x_{k_{p}^{* *}}\right|\right)+\hat{\sigma}(\bar{d})
\end{aligned}
$$

for some $\mathcal{K}_{\infty}$-function $\hat{\alpha}_{3}(\cdot)$ and some $\mathcal{K}$-function $\hat{\sigma}(\cdot)$. To this end, let us introduce two properties of comparison functions that will be used in the proof. Given a $\mathcal{K}_{\infty}$-function $\gamma(\cdot)$, for any pair $\left(s^{\prime}, s^{\prime \prime}\right) \in \mathbb{R}_{\geq 0} \times \mathbb{R}_{\geq 0}$ it holds that

$$
\begin{aligned}
\gamma\left(s^{\prime}\right) & =\gamma\left(s^{\prime}+s^{\prime \prime}-s^{\prime \prime}\right) \\
& \leq \gamma\left(s^{\prime \prime}+\left|s^{\prime}-s^{\prime \prime}\right|\right) \\
& \leq \gamma\left(\max \left\{2 s^{\prime \prime}, 2\left|s^{\prime}-s^{\prime \prime}\right|\right\}\right) \\
& \leq \gamma\left(2 s^{\prime \prime}\right)+\gamma\left(2\left|s^{\prime}-s^{\prime \prime}\right|\right),
\end{aligned}
$$

and also

$$
-\gamma\left(2 s^{\prime \prime}\right) \leq-\gamma\left(s^{\prime}\right)+\gamma\left(2\left|s^{\prime}-s^{\prime \prime}\right|\right)
$$

Now, let us derive a series of intermediate bounding inequalities. In view of Assumption 4, the following bound holds:

$$
\begin{aligned}
V\left(\hat{x}_{k_{p}^{* *}}\right) & \leq V\left(x_{k_{p}^{* *}}\right)+\gamma\left(\left|x_{k_{p}^{* *}}-\hat{x}_{k_{p}^{* *}}\right|\right) \\
& \leq V\left(x_{k_{p}^{* *}}\right)+\gamma\left(\mathcal{G}_{\gamma_{\bar{\tau} r t}}(\bar{d})\right),
\end{aligned}
$$

which yields

$$
-V\left(x_{k_{p}^{* *}}\right) \leq-V\left(\hat{x}_{k_{p}^{* *}}\right)+\gamma\left(\mathcal{G}_{\gamma_{\bar{\tau} r t}}(\bar{d})\right)
$$

Moreover, in view of the following two inequalities

$$
\begin{aligned}
V\left(F\left(x_{k_{p}^{* *}}, u_{k_{p}^{* *}}\right)\right) & \leq V\left(\hat{F}\left(\hat{x}_{k_{p}^{* *}}, u_{k_{p}^{* *}}\right)+d_{k_{p}^{* *}}\right) \\
& +\gamma\left(\left|\hat{F}\left(\hat{x}_{k_{p}^{* *}}, u_{k_{p}^{* *}}\right)-F\left(x_{k_{p}^{* *}}, u_{k_{p}^{* *}}\right)\right|+\bar{d}\right)
\end{aligned}
$$

and

$$
\begin{aligned}
& \left|\hat{F}\left(\hat{x}_{k_{p}^{* *}}, u_{k_{a}}\right)-F\left(x_{k_{p}^{* *}}, u_{k_{p}^{* *}}\right)\right| \leq \\
& \leq\left|\hat{F}\left(\hat{x}_{k_{p}^{* *},} u_{k_{a}}\right)-\hat{F}\left(x_{k_{p}^{* *}}, u_{k_{p}^{\prime \prime}}\right)\right| \\
& +\left|\hat{F}\left(x_{k_{p}^{* *}}, u_{k_{p}^{* *}}\right)-F\left(x_{k_{p}^{* *}}, u_{k_{p}^{* *}}\right)\right| \\
& \leq \eta\left(\mathcal{G}_{\eta_{\bar{\tau}_{r t}}}(\bar{d})\right)+\bar{d} \\
& =\mathcal{G}_{\eta_{\bar{\tau}_{r t}+1}}(\bar{d}) \text {, }
\end{aligned}
$$

we have that

$$
\begin{aligned}
V\left(F\left(x_{k_{p}^{* *}}, u_{k_{p}^{* *}}\right)\right) \leq & V\left(\hat{F}\left(\hat{x}_{k_{p}^{* *}}, u_{k_{p}^{* *}}\right)+d_{k_{p}^{* *}}\right) \\
& +\gamma\left(2 \mathcal{G}_{\eta_{\bar{\tau}_{r t}+1}}(\bar{d})\right)+\gamma(2 \bar{d}),
\end{aligned}
$$

where we have used the property (31) of comparison functions. In view of (33), (34), (12) and (25), and by using the property (32), we obtain

$$
\begin{aligned}
& V\left(F\left(x_{k_{p}^{* *}}, u_{k_{p}^{* *}}\right)\right)-V\left(x_{k_{p}^{* *}}\right) \leq \\
& \leq V\left(\hat{F}\left(\hat{x}_{k_{p}^{* *}}, \kappa^{*}\left(\hat{x}_{k_{p}^{* *}}\right)\right)+d_{k_{p}^{* *}}\right)-V\left(\hat{x}_{k_{p}^{* *}}\right) \\
&+\gamma\left(2 \mathcal{G}_{\eta_{\bar{\tau}_{r t}+1}}(\bar{d})\right)+\gamma\left(\mathcal{G}_{\eta_{\bar{\tau}_{r t}}}(\bar{d})\right)+\gamma(2 \bar{d}) \\
& \leq-\alpha_{3}\left(\left|\hat{x}_{k_{p}^{* *}}\right|\right)+\sigma(\bar{d})+\gamma\left(2 \mathcal{G}_{\eta_{\bar{\tau}_{r t}+1}}(\bar{d})\right) \\
&+\gamma\left(\mathcal{G}_{\eta_{\bar{\tau}_{r t}}}(\bar{d})\right)+\gamma(2 \bar{d})
\end{aligned}
$$

Now, applying (32) with $\alpha_{3}(\cdot)$ in place of $\gamma(\cdot), s^{\prime}=$ $\frac{1}{2}\left|x_{k_{p}^{* *}}\right|$ and $s^{\prime \prime}=\frac{1}{2}\left|\hat{x}_{k_{p}^{* *}}\right|$ it holds that

$$
-\alpha_{3}\left(\left|\hat{x}_{k_{p}^{* *}}\right|\right) \leq-\alpha_{3}\left(\frac{1}{2}\left|x_{k_{p}^{* *}}\right|\right)+\alpha_{3}\left(\left|x_{k_{p}^{* *}}-\hat{x}_{k_{p}^{* *}}\right|\right)
$$

which becomes, using (25)

$$
-\alpha_{3}\left(\left|\hat{x}_{k_{p}^{* *}}\right|\right) \leq-\alpha_{3}\left(\frac{1}{2}\left|x_{k_{p}^{* *}}\right|\right)+\alpha_{3}\left(\Gamma_{\eta_{\bar{\tau} r t}}(\bar{d})\right)
$$

Finally, taking into account (36), (35) can be further upper bounded by

$$
\begin{aligned}
& V\left(F\left(x_{k_{p}^{* *}}, u_{k_{p}^{* *}}\right)\right)-V\left(x_{k_{p}^{* *}}\right) \leq \\
& \leq-\alpha_{3}\left(\frac{1}{2}\left|x_{k_{p}^{* *}}\right|\right)+\alpha_{3}\left(\Gamma_{\eta_{\bar{\tau}_{r t}}}(\bar{d})\right)+\sigma(\bar{d}) \\
& \quad+\gamma\left(2 \mathcal{G}_{\eta_{\bar{\tau}_{r t}+1}}(\bar{d})\right)+\gamma\left(\mathcal{G}_{\eta_{\bar{\tau}_{r t}}}(\bar{d})\right)+\gamma(2 \bar{d})
\end{aligned}
$$

which implies that (30) holds with

$$
\hat{\alpha}_{3}(s)=\alpha_{3}\left(\frac{1}{2} s\right), \forall s \in \mathbb{R}_{\geq 0},
$$

and

$$
\begin{aligned}
\hat{\sigma}(s)= & \alpha_{3}\left(\Gamma_{\eta_{\bar{\tau}_{r t}}}(s)\right)+\sigma(s)+\gamma\left(2 \mathcal{G}_{\eta_{\bar{\tau}_{r t}+1}}(s)\right) \\
& +\gamma\left(\mathcal{G}_{\eta_{\bar{\tau}_{r t}}}(s)\right)+\gamma(2 s), \quad \forall s \in \mathbb{R}_{\geq 0} .
\end{aligned}
$$

Finally, the confinement in $X$ can be proven by evaluating the ISS-asymptotic gains induced by the $\mathcal{K}$-functions of the ISSLyapunov inequalities. Let us define $\alpha_{4}(s)=\hat{\alpha}_{3}\left(\alpha_{2}^{-1}(s)\right)$ and pick $\hat{\alpha}_{4}$ as in Lemma 2.4 of [46]. Letting $c$ be any number in $(0,1)$ and $\hat{\eta}(s)=\hat{\alpha}_{4}^{-1}(\hat{\sigma} / c)$, then the asymptotic gain is given by

$$
\eta(s)=\alpha_{1}^{-1}(\hat{\eta}(s)) \text {. }
$$

Moreover

$$
\beta(s, r)=\alpha_{1}^{-1}\left(\hat{\beta}\left(\alpha_{2}(s)\right), r\right),
$$


where $\hat{\beta}(s, r)=z(r)$ is the solution of the scalar difference equation

$$
z(r+1)=z(r)-(1-c) \hat{\alpha}_{4}(z(r)),
$$

with $z(0)=s$. It holds that

$$
\beta(s, 0)=\alpha_{1}^{-1}\left(\hat{\beta}\left(\alpha_{2}(s)\right)\right) .
$$

Let $\beta_{0}(s) \triangleq \beta(s, 0)$. Since $|x(k, \bar{x}, \mathbf{d})| \leq \beta(|\bar{x}|, k)+$ $\eta(|| \mathbf{d}||) \leq \beta_{0}(|\bar{x}|)+\eta(\| \mathbf{d}||)$, then

$$
\beta_{0}(|\bar{x}|)+\eta(\bar{d}) \leq \underline{X} \Longrightarrow x(k, \bar{x}, \mathbf{d}) \in X,
$$

with $\underline{X} \triangleq d_{h}\left(\{0\}, \mathbb{R}^{n} \backslash X\right)$ and where $\mathrm{d}_{h}$ denotes the Haussdorf distance between sets ${ }^{1}$.

Therefore, for any $\bar{d}<\eta^{-1}(\underline{X})$, requiring that $|\bar{x}| \leq$ $\beta_{0}^{-1}(\underline{X}-\eta(\bar{d}))$, the confinement of the trajectories in the modeling domain $X$ is guaranteed. Then for any $\bar{d}: 0<$ $\bar{d}<\eta^{-1}(\underline{X})$ the set of admissible initial conditions

$$
\Xi_{0} \triangleq\left\{\xi \in \mathbb{R}^{n_{x}}:|\xi| \leq \beta_{0}^{-1}(\underline{X}-\eta(\bar{d}))\right\} .
$$

for which regional-ISS is preserved is non-empty.

To sum up, Theorem 5.1 paves the way to design controlled networked control systems that are robustly stable when nominally-stabilising control laws are embedded into a networked context thanks to the proposed policy.

\section{Simulation Results}

In this section, two simulation use cases are presented. The first one deals with networked control of a continuous stirred tank reactor and aims at comparing the proposed methodology with a different technique reported in the literature. The second use case addresses a challenging networked control problem for a VTOL quadcopter.

\section{A. Networked Control of a Stirred Tank Reactor: Comparative Results}

In the literature, several application examples can be found in which the effectiveness of specific design approaches of control systems over a communication network is shown. For fair comparison purposes, we consider the networked control technique presented in [47], where a Lyapunov-based model predictive control (LMPC) technique for nonlinear systems subject to time-varying communication delays between the plant (measurement sensors) and the controller is presented and evaluated. In this section, we compare this technique with the proposed NMPC \& MBSF-PCS strategy using the same testbed, namely a well mixed, non-isothermal continuous stirred tank reactor described in detail in [48]:

$$
\begin{aligned}
& \frac{d T}{d t}=\frac{F}{V_{r}}\left(T_{A 0}-T\right)-\sum_{i=1}^{3} \frac{\Delta H_{i}}{\sigma c_{p}} k_{i 0} \mathrm{e}^{\frac{-E_{i}}{R T}} C_{A}+\frac{Q}{\sigma c_{p} V_{r}} \\
& \frac{d C_{A}}{d t}=\frac{F}{V_{r}}\left(C_{A 0}+\Delta C_{A 0}-C_{A}\right)-\sum_{i=1}^{3} k_{i 0} \mathrm{e}^{\frac{-E_{i}}{R T}} C_{A} .
\end{aligned}
$$

\footnotetext{
${ }^{1}$ In the present case, $\underline{X}$ is the radius of the largest hyper-sphere centered in the origin and contained in $X$.
}
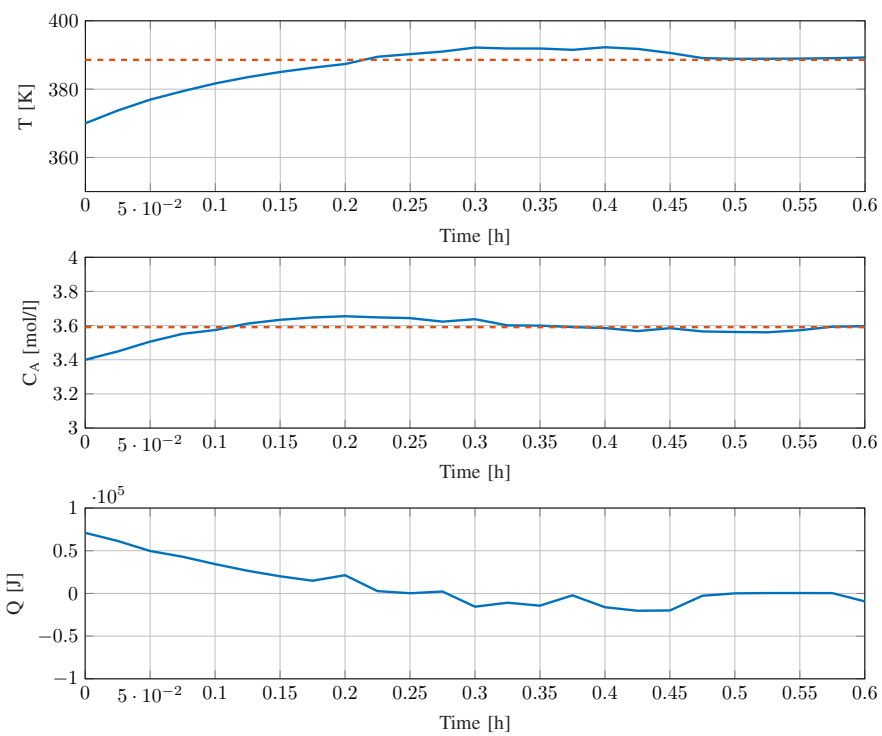

Fig. 2: Trajectory of the system and corresponding input using the MBSF in the same configuration of [47].

The states of the system are the temperature of the reactor $T$

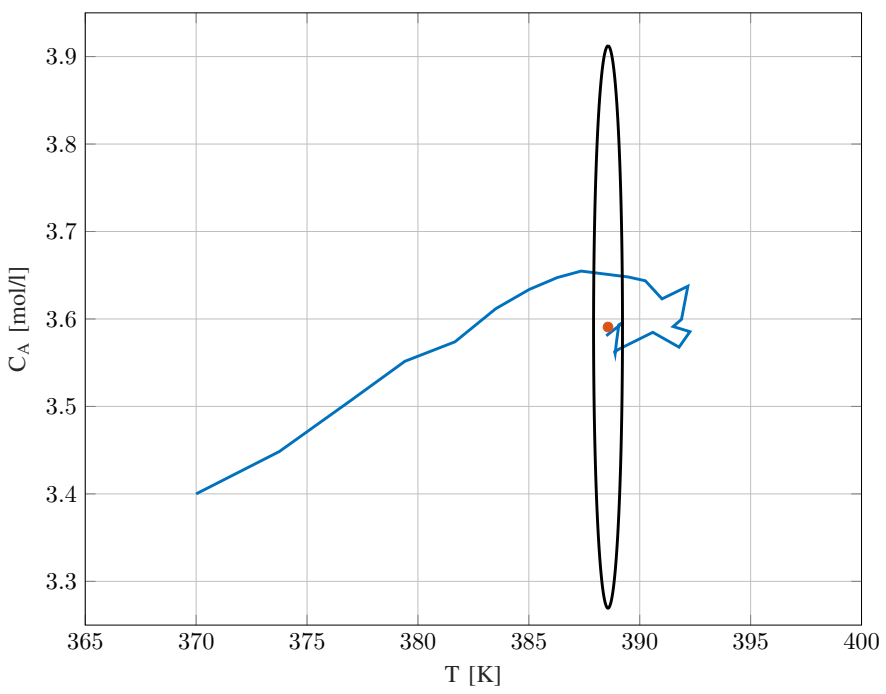

Fig. 3: Trajectory of the system using the MBSF (same configuration of [47]). Note that the system reaches the terminal set constraint despite the delays in the down-link.

and the molar concentration $C_{A}, Q$ is the input to the system (heat supplied or removed to the reactor, $|Q| \leq 10^{5} \mathrm{~kJ} / \mathrm{h}$ ) and $\Delta C_{A 0}$ is an unknown, bounded time-varying uncertainty $\left(\left|\Delta C_{A 0}\right| \leq 0.2 \mathrm{~mol} / \mathrm{l}\right)$. The aim of the control is to stabilize the open-loop unstable steady state $T_{s}=388 \mathrm{~K}$ and $C_{A, s}=$ $3.59 \mathrm{~mol} / \mathrm{l}$. In order to adequately compare the two different NCSs, the parameters of the controller and of the system are the same of [47], that is:

- sampling time: $\Delta=0.025 \mathrm{~h}$;

- maximum transmission delay in the down-link: $\bar{\tau}_{s c}=6$;

- maximum transmission delay in the up-link: $\bar{\tau}_{c a}=0$ (same as [47], that is, ideal up-link connection); 
- prediction horizon of the MPC controller: $N_{p}=7$;

- MPC cost function:

$$
\sum_{i=0}^{N_{c}} x_{t_{i}}^{T} Q_{c} x_{t_{i}}+u_{t_{i}}^{T} R_{c} u_{t_{i}}
$$

with $Q_{c}=\operatorname{diag}\left(1,10^{4}\right), R_{c}=10^{-6}$.
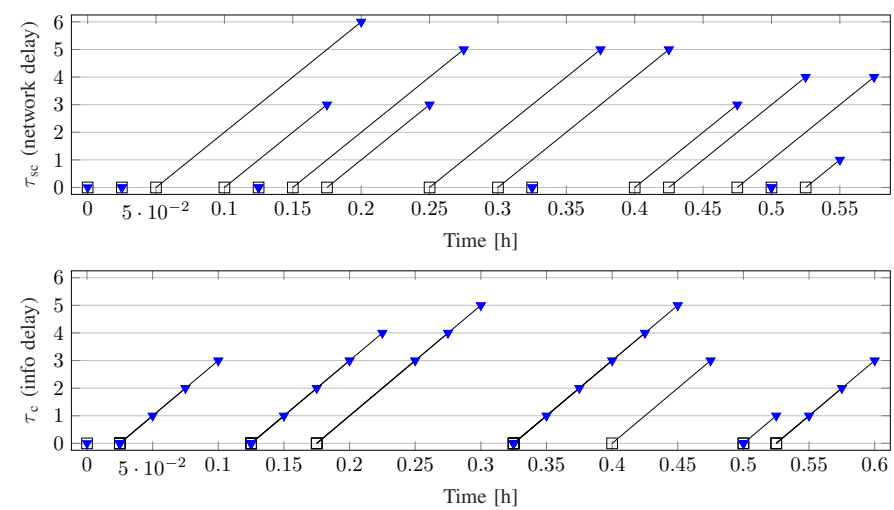

Fig. 4: Delays and packet losses in the down-link (same configuration of [47]). The upper diagram represents the network induced delays: squares correspond to sending instants and triangles to receiving instants. In the bottom diagram the age of the information used by the controller (corresponding to triangles) is represented. Since each packet can be used multiple times (in case of transmission delay) there can be more than one triangle on the same slanted segment.
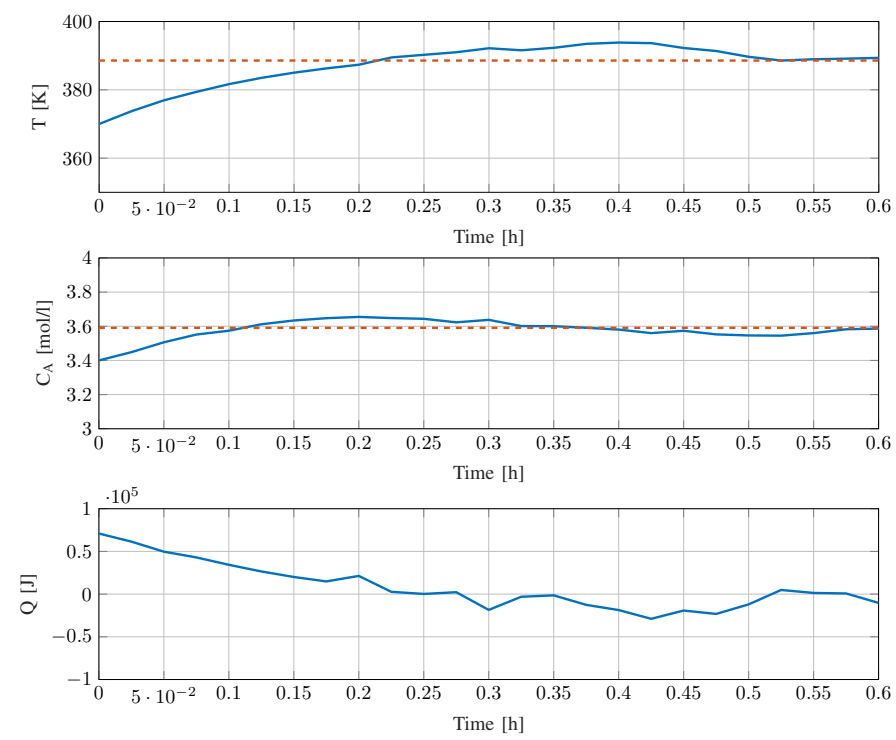

Fig. 5: Trajectory of the system and corresponding input using the MBSF with delays and packet losses in both down-link and up-link

In Fig. 2 the state evolution over time and the input to the system are represented, whereas in Fig. 3 the state trajectory and the terminal set of the MPC are shown. During its evolution, the system is subjected to time delays represented

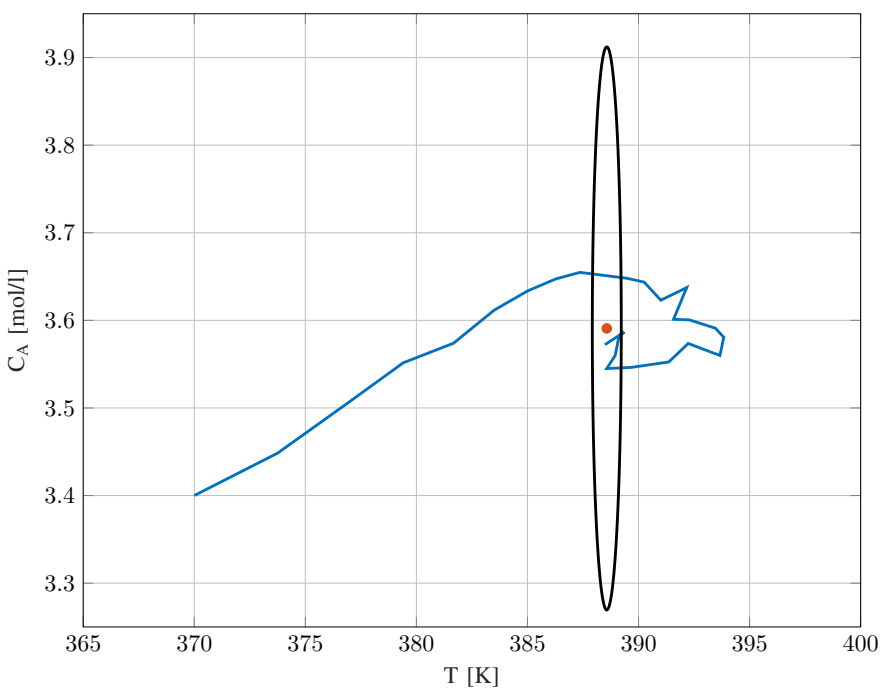

Fig. 6: Trajectory of the system using the MBSF (delays and packet losses in both down-link and up-link).

pictorially in Fig. 4, where the timing diagrams of the simulated networked packet-based communication links are given. As it can be noticed, the state variables converge to their set points (represented in red) while the input decays to zero; hence in this case performances of the proposed control policy are similar to the ones presented in Fig. 5 of [47].

Since the control scheme proposed in the present paper is able to handled delays and data losses in both the up-link and the down-link (unlike the one of [47]), the effectiveness of the controller is evaluated also in this more challenging configuration. Simulations have been carried out using the following parameters:

- maximum transmission delay in the down-link: $\bar{\tau}_{s c}=2$;

- maximum transmission delay in the up-link: $\bar{\tau}_{c a}=1$;

- bounds on the maximum number of consecutive dropouts in the up-link and in the down-link, respectively: $\bar{L}_{s c}=1$, $\bar{L}_{c a}=2$.

In Figs. 5 and 6 the state trajectories are shown as well as the input actions applied to the system obtained when the disturbance variable is set to $\left|\Delta C_{A 0}\right| \leq 0.2 \mathrm{~mol} / \mathrm{l}$ (same as in the previous simulation case), that, in turn, corresponds to:

$$
\bar{d}=\frac{F}{V_{r}}\left|\Delta C_{A 0}\right|=1.00 \mathrm{~mol} /(\mathrm{h} \cdot \mathrm{l}) .
$$

Delays and packet drops are represented in Fig. 7. Simulation results show that taking into account the non-ideal communication even in the up-link does not cause a large deterioration of the performances.

Remark 6.1: The simulation scenario considered above shows that the proposed method allows for bounds on the uncertainties that are not too conservative thus confirming the practical robustness of the control scheme. In this respect, it is worth remarking that the theoretical estimate of the norm of the maximum tolerable additive disturbance may result to be very conservative. Specifically, in the present example, the method explained in [35] provides the estimate $\bar{d} \leq 4.85 \times 10^{-21} \mathrm{~mol} /(\mathrm{h} \cdot 1)$. This very conservative estimate 
has only a theoretical interest but has no impact on the practical design of the controller.
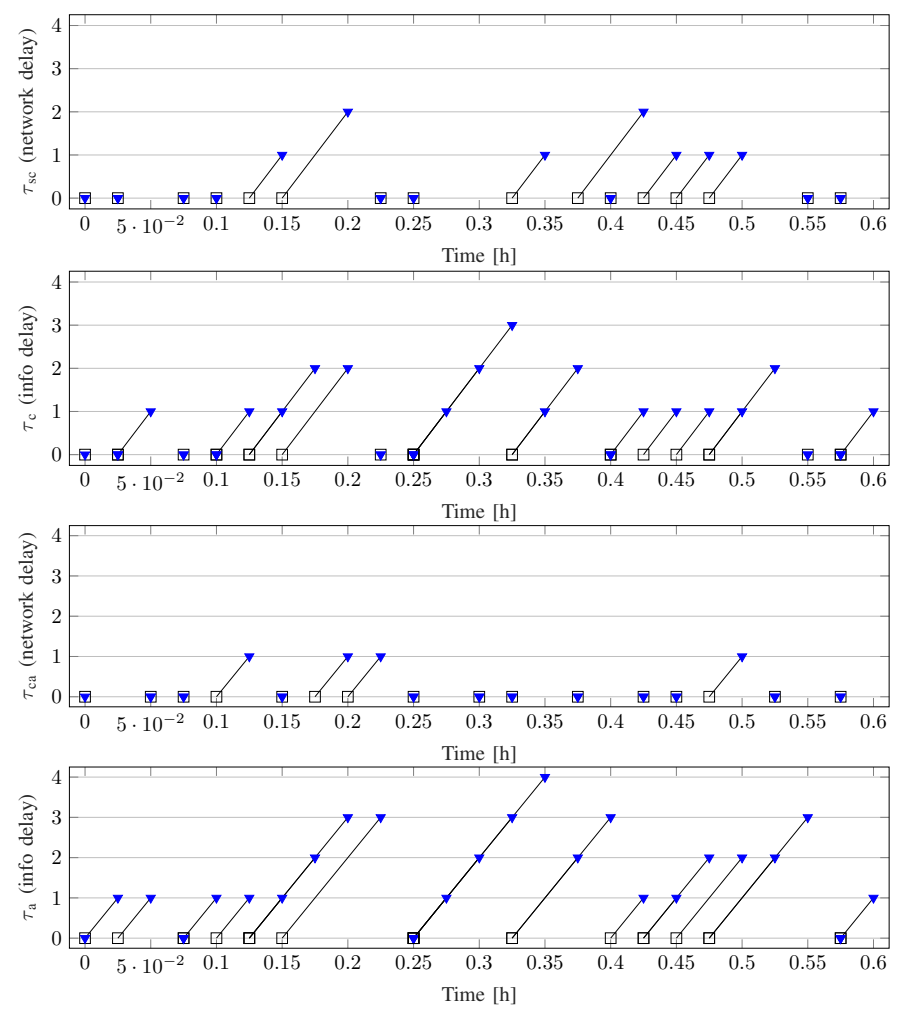

Fig. 7: Delays and packet losses in the down-link and uplink. $\bar{\tau}_{c a}$ and $\bar{\tau}_{a}$ diagrams are referred to the up-link (in this simulation packet losses and transmission delays are present in both up-link and down-link).

Finally, in order to quantitatively compare the MBSF policy with the LMPC, the same performance index of [47] has been defined:

$$
\sum_{i=0}^{M} x_{t_{i}}^{T} Q_{c} x_{t_{i}}+u_{t_{i}}^{T} R_{c} u_{t_{i}}
$$

where the final simulation time $t_{M}$ has been set to $2 \mathrm{~h}$. Then a total number of 100 simulations under the same conditions of [47] (that is, $N_{p}=10$ and initial state randomly chosen inside their stability region) have been carried out. The mean of the cost obtained for each simulation is $1.0039 \times 10^{4}$, while the average cost obtained by Liu et al. $^{2}$ is $1.7364 \times 10^{4}$. Hence, although the controller designed by Liu et al. takes into account only delays in the down-link, this result shows that the MBSF policy, besides being more generic (since it allows to cope with delays and packet dropouts in both the up-link and down-link), achieves performances comparable to the LMPC designed in [47].

\section{B. Networked Control of a VTOL Quadcopter}

Let us consider a VTOL (Vertical Take-Off and Landing) quadcopter, modelled as a six degrees of freedom rigid body. In particular, we refer to the mathematical model of the OS4

${ }^{2}$ This is the mean value of the first column of table II of [47] quadcopter, described in detail in [49] (see also [50], [51], [52] for different attitude and altitude control strategies applied to the OS4 prototype). The dynamics of such device is usually described by means of a nonlinear system, using the following 12 state variables:

$$
X=[\phi, \dot{\phi}, \theta, \dot{\theta}, \psi, \dot{\psi}, z, \dot{z}, x, \dot{x}, y, \dot{y}]^{T}
$$

where, as usual, $\phi, \theta, \psi$ are respectively the roll, pitch and yaw angles, whereas the $x, y, z$ are the coordinates of the drone's center of the mass. For the sake of brevity, we are reporting only the state vector and the dynamics of the OS4 device, referring to [49] (in particular to Chapters 2 and 4) for a detailed description of the OS4 model equations:

$$
\frac{d X}{d t}=\left[\begin{array}{c}
\dot{\phi} \\
\dot{\theta} \dot{\psi}+\dot{\theta} a_{2} \Omega_{r}+b_{1} U_{2} \\
\dot{\theta} \\
\dot{\phi} \dot{\psi} a_{3}-\dot{\phi} a_{4} \Omega_{r}+b_{2} U_{3} \\
\dot{\psi} \\
\dot{\theta} \dot{\phi} a_{5}+b_{3} U_{4} \\
\dot{z} \\
g-(\cos (\phi) \cos (\theta)) \frac{U_{1}}{m} \\
\dot{x} \\
u_{x} \frac{U_{1}}{m} \\
\dot{y} \\
u_{y} \frac{U_{1}}{m}
\end{array}\right]
$$

with

$$
\begin{aligned}
& u_{x}=(\cos (\phi) \sin (\theta) \cos (\psi)+\sin (\phi) \sin (\psi)) \\
& u_{y}=(\cos (\phi) \sin (\theta) \sin (\psi)-\sin (\phi) \cos (\psi))
\end{aligned}
$$

The variables $U_{1}, \ldots, U_{4}$ denote the inputs to the system ( $U_{1}, U_{2}, U_{3}$ are forces, whereas $U_{4}$ is a torque). It is worth noting that the variable $U_{1}$ is related to the overall thrust available using the four available drives, whereas the variables $U_{2}, U_{3}, U_{4}$ are related to the roll, pitch and yaw torques, respectively. ${ }^{3}$

In the present simulation study, we assume that the quadcopter attitude perturbations from the hovering condition are sufficiently small to allow considering the rates of change of the orientation angles to be equal to the body angular velocity components (in the general case, a non-linear transformation matrix is needed to obtain the body angular velocities from the rates of change of the orientation angles). This assumption has been made also in [49], [50], [51], [52]. The performance index to be minimized is defined as follows:

$$
J=\sum_{i=0}^{N-1}\left[X_{t_{i}}^{T} Q_{c} X_{t_{i}}+U_{t_{i}}^{T} R_{c} U_{t_{i}}\right]+a X_{t_{N}}^{T} P X_{t_{N}},
$$

where $X_{t_{i}}$ is the full state vector as of Eq. (40) at time instant $t_{i}$ and $U_{t_{i}}=\left[U_{1}\left(t_{i}\right), U_{2}\left(t_{i}\right), U_{3}\left(t_{i}\right), U_{4}\left(t_{i}\right)\right]^{T}$. The penalty coefficient $a$ and the matrix $P$ of the final cost term have been properly tuned according to the constructive procedure

\footnotetext{
${ }^{3}$ The reader is referred to [49] (Chapters 2 and 4) for the detailed description and explanation of the dynamic state equations. Moreover, the OS4 parameters' values are reported in [49] (Appendix E).
} 


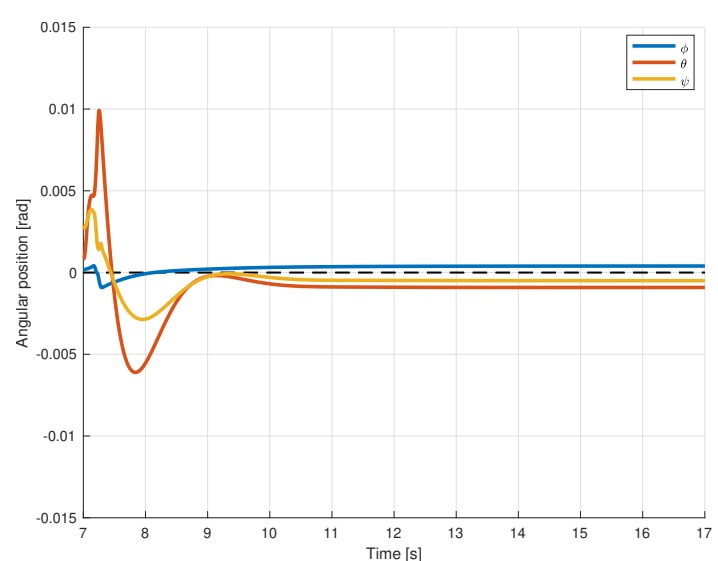

(a) roll, pitch and yaw angles

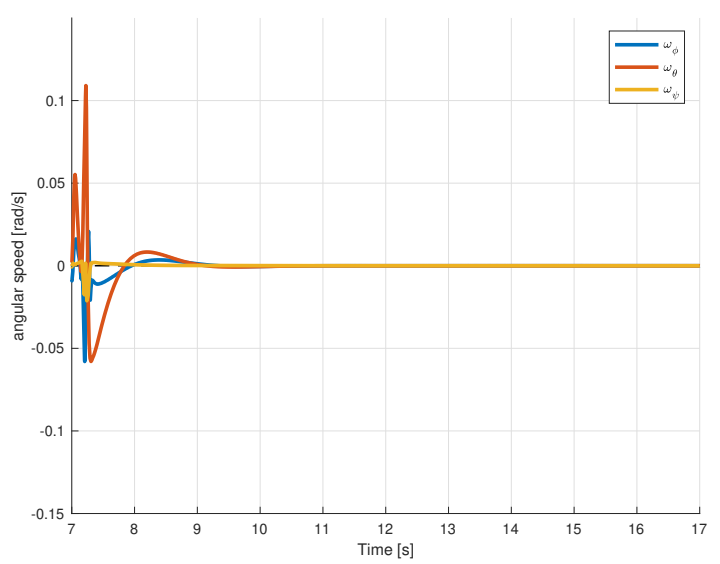

(c) angular speeds

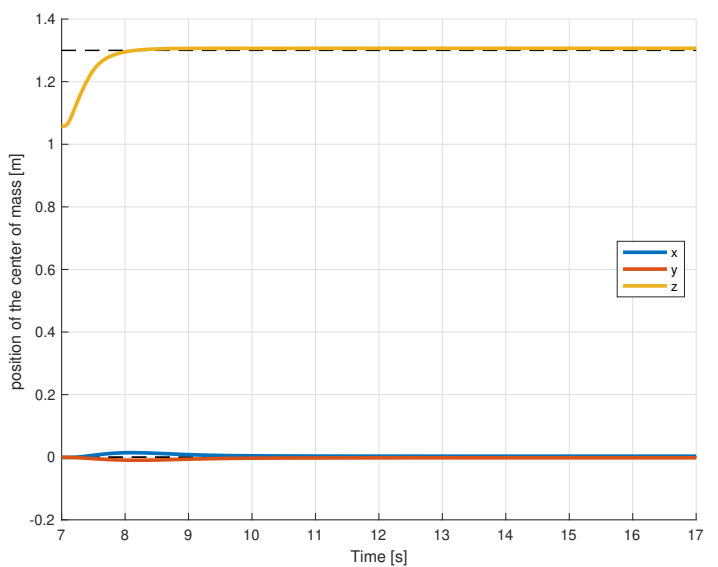

(b) coordinates of the center of mass

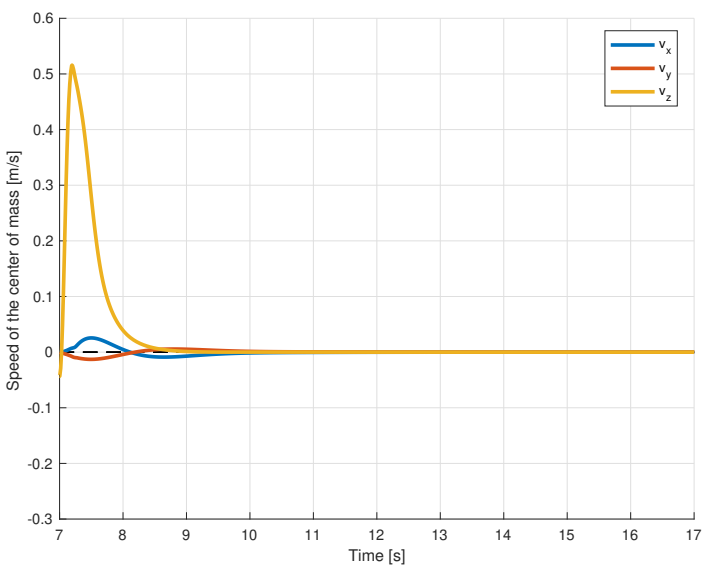

(d) speed components of the center of mass

Fig. 8: VTOL example - Perfect situation: no transmission delays, no packet dropouts, no disturbances. The dashed lines correspond to the reference values.

presented in [53]. Finally,

$$
\begin{aligned}
Q_{c}= & \operatorname{diag}\left(10^{5}, 10^{5}, 10^{5}, 10^{5}, 10^{5}, 10^{5},\right. \\
& \left.10^{-1}, 10^{-1}, 10^{-1}, 10^{-1}, 10^{-1}, 10^{-1}\right) \\
R_{c}= & \operatorname{diag}\left(2.55 \cdot 10^{8}, 5.10 \cdot 10^{8}, 5.10 \cdot 10^{8}, 4.44 \cdot 10^{11}\right) .
\end{aligned}
$$

Applying the $Q_{c}$ matrix in the performance index to be minimized by the NMPC strategy, we would design a control law able to keep as constant as possible the attitude of the VTOL; moreover the weight matrix for the input signals $U_{i}$ is taking into account the different inertia values on the main axes of inertia (please, refer to Appendix E in [49] for the values): the inertia on the $x$ and $y$ axes (in the body frame) are similar, but the inertia on the $z$ axis is quite different. This is the motivation why the weight for $U_{4}$ is different from the weights for $U_{2}$ and $U_{3}$.

Figs. 8 and 9 illustrate the application of the NMPC in two different conditions: without transmission delays and packet drops (a perfectly efficient transmission network) and without any uncertainty on the model in the first case, and with some transmission delays, data losses and uncertainties on the model equations (in particular, only the angular and translational acceleration components have been considered as affected by uncertainty) in the second situation. Each figure depicts the temporal evolution of the roll, pitch and yaw angles, the coordinates of the drone center of mass, the body angular speeds and the center of mass speed components. In the latter case, the MBSF policy has been applied and Fig. 10 describes the transmission of data packets from sensors to the controller and then from the controller to the actuators, and how the information of each packet has been used according to the MBSF strategy.

In both the simulations, for the take-off and during the first 7 seconds of the fly the OS4 model has been controlled using a standard control strategy (namely the integral backstepping described in [52], [49]): in this way the drone reaches an altitude higher than 1.0 meter above the ground (this part of the simulation is not depicted in the figures). Then in both the scenarios the NMPC control strategy (with the MBSF policy in the latter scenario) has been activated, with as target the altitude of 1.3 meters above the ground while keeping the hovering attitude. When the MBSF-PCS policy has been applied, also additive uncertainties (with proper bound $\bar{d}$ ) have been injected to the components of the state transition function, describing the time evolution of angular and translational acceleration components.

For the simulations the following data have been used:

- sampling time: $\Delta=20.0 \cdot 10^{-3} \mathrm{~s}$ 


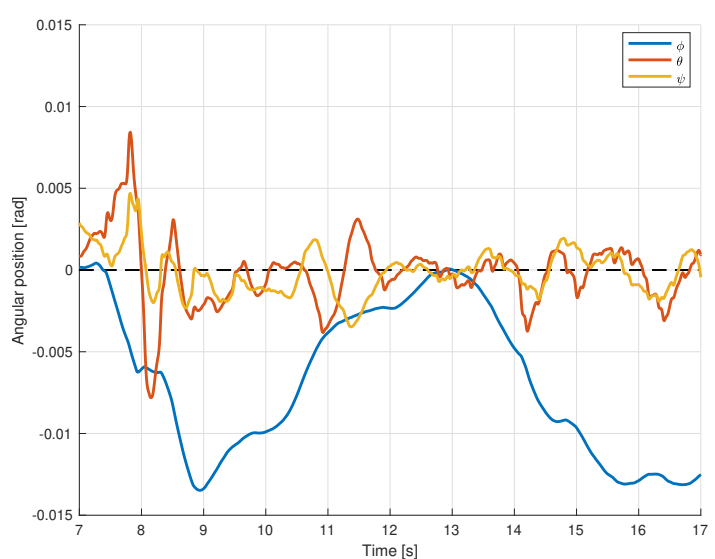

(a) roll, pitch and yaw angles

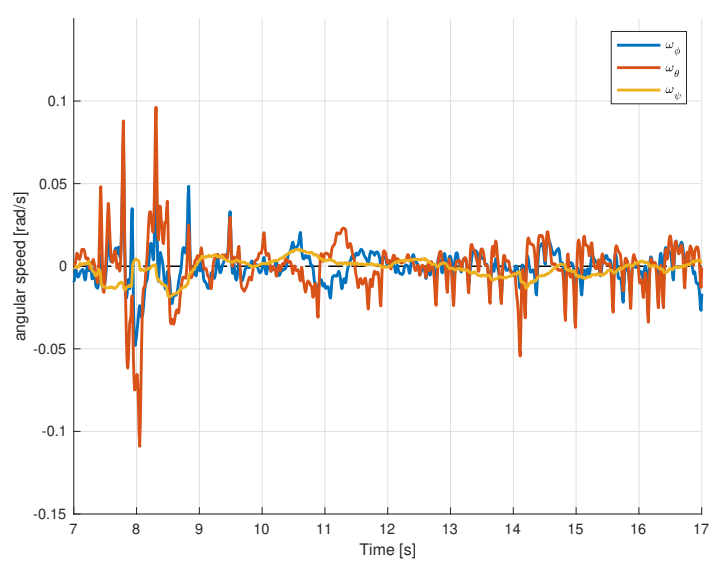

(c) angular speeds

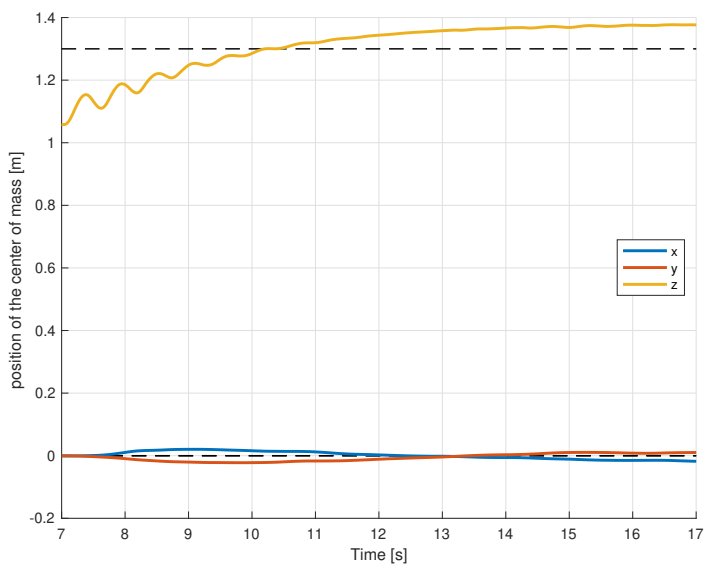

(b) coordinates of the center of mass

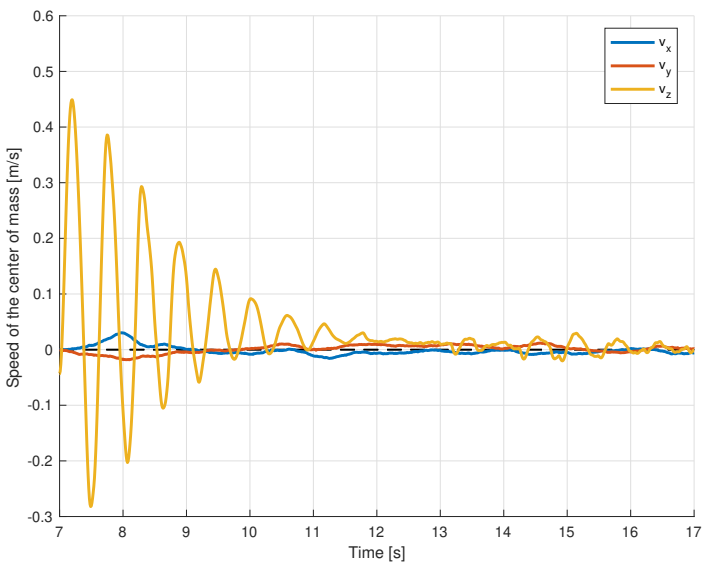

(d) speed components of the center of mass

Fig. 9: VTOL example - The case with transmission delays, packet dropouts and uncertanty on angular and translational acceleration components (bound on uncertainty $\bar{d}=3.8 \cdot 10^{-4}$ ). The dashed lines correspond to the reference values.
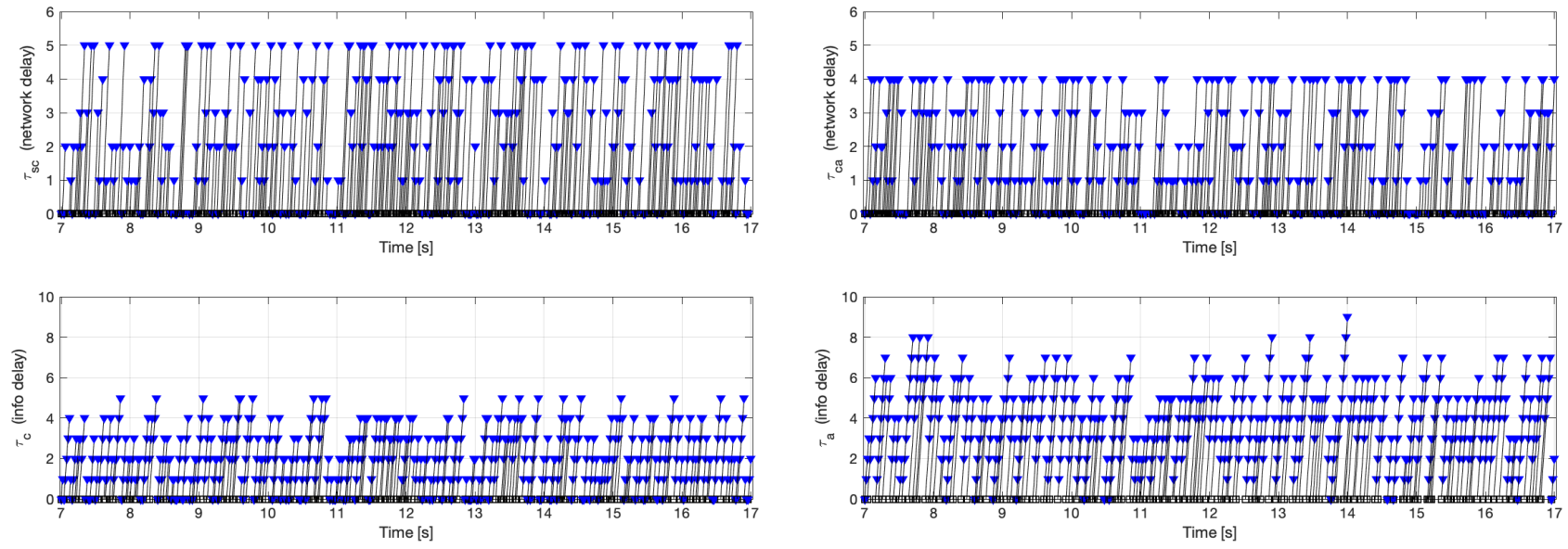

(a) Arrival times and use of each packet according to the MBSF-PCS policy (b) Arrival times and use of each packet according to the MBSF-PCS policy in the down-link. in the up-link.

Fig. 10: VTOL example - Delays and packet losses in the down-link (a) and up- link (b). 

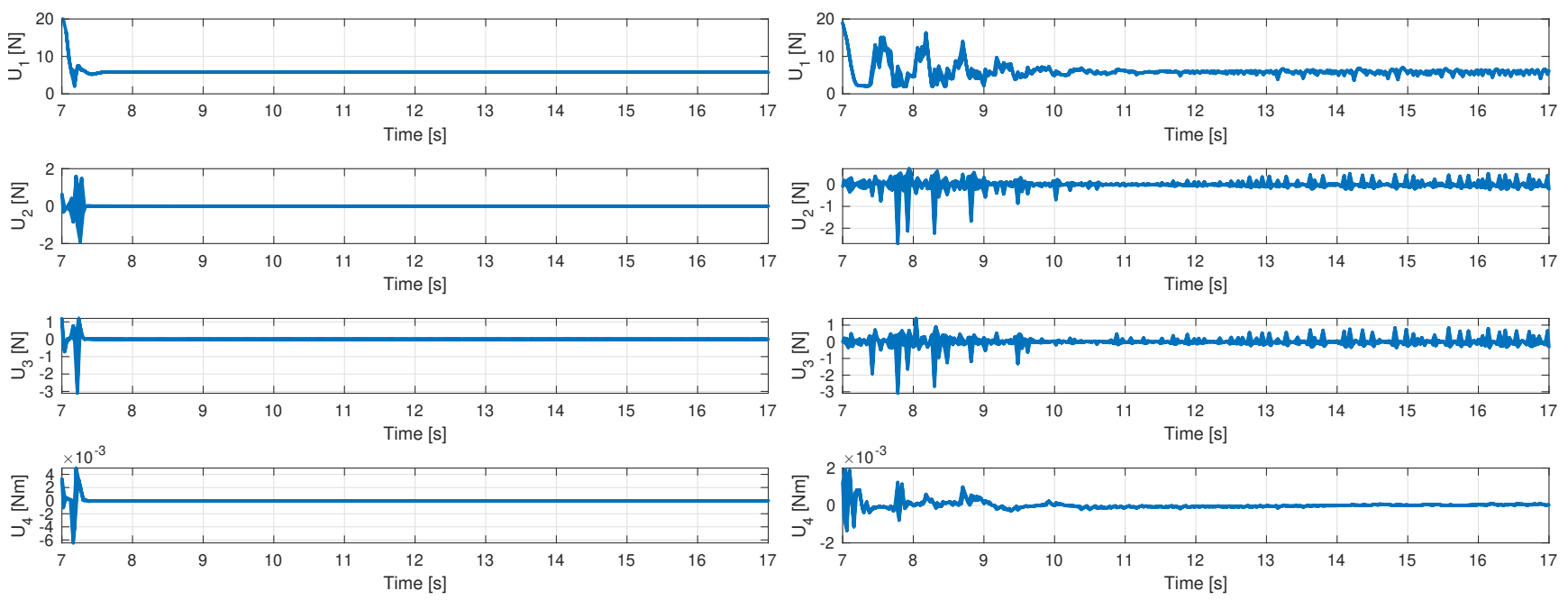

(a) VTOL input signals in case of perfect scenario

(b) VTOL input signals in case of transmission network with delays, dropouts and disturbances acting on the system

Fig. 11: VTOL input signals: on the left side the case of no disturbances and perfect transmission networks, on the right part the case when transmission delays and dropouts are considered and also disturbances are acting on the system (bound on uncertainty $\bar{d}=3.8 \cdot 10^{-4}$ ).

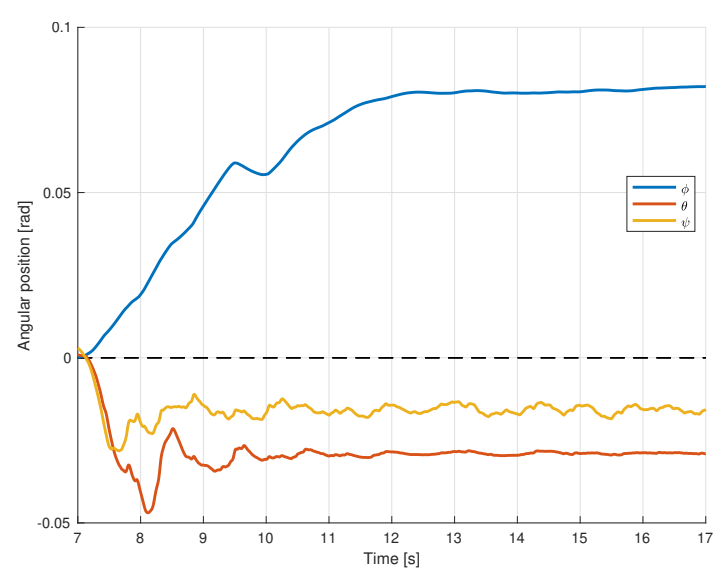

(a) roll, pitch and yaw angles

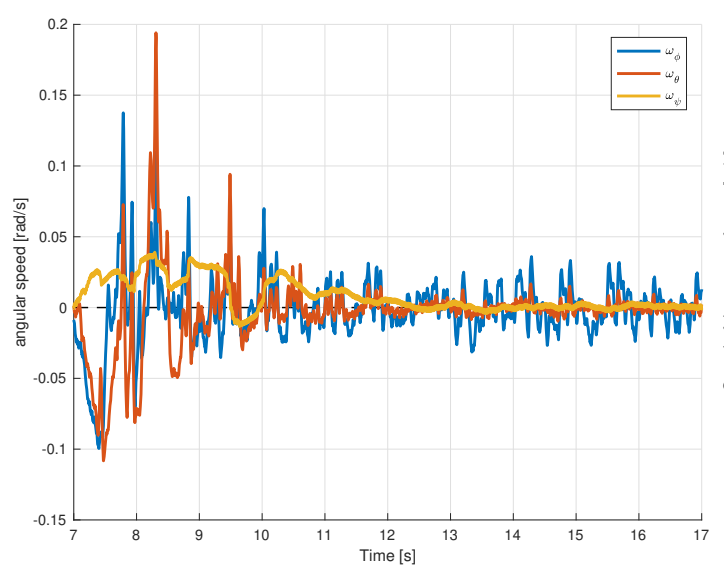

(c) angular speeds

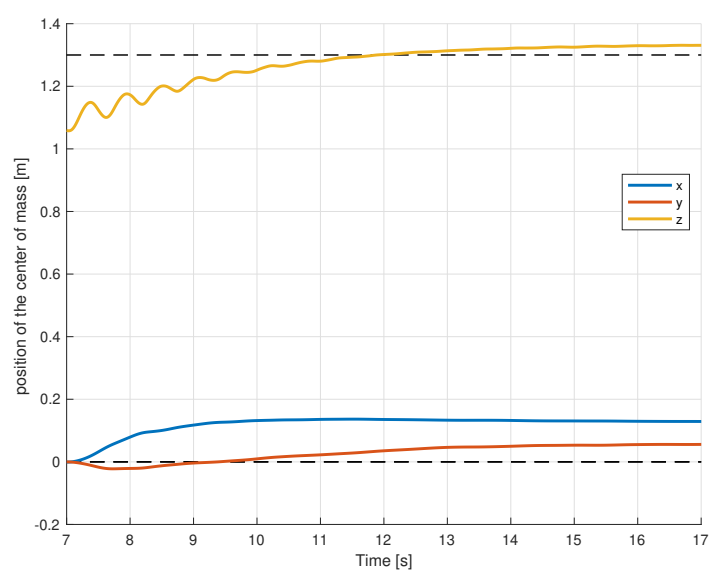

(b) coordinates of the center of mass

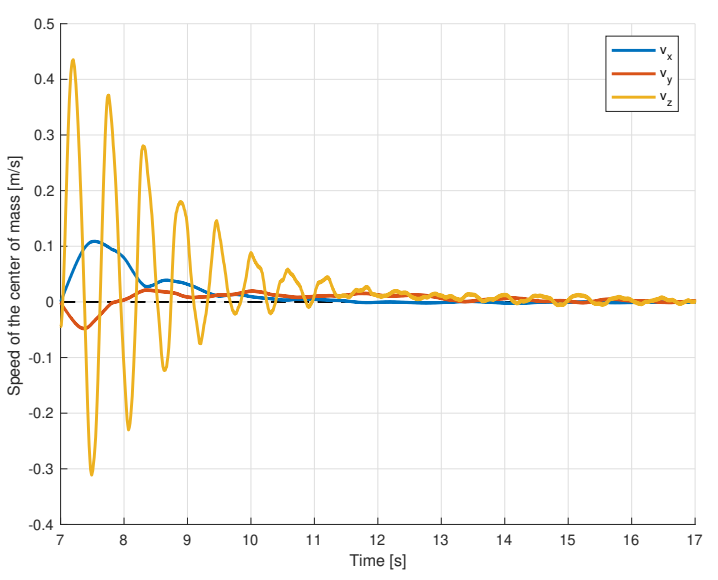

(d) speed components of the center of mass

Fig. 12: VTOL example - The case with transmission delays, packet dropouts and uncertanty on angular and translational acceleration components (bound on uncertainty $\tilde{d}=1.0 \cdot 10^{-1}$ ). The dashed lines correspond to the reference values. 

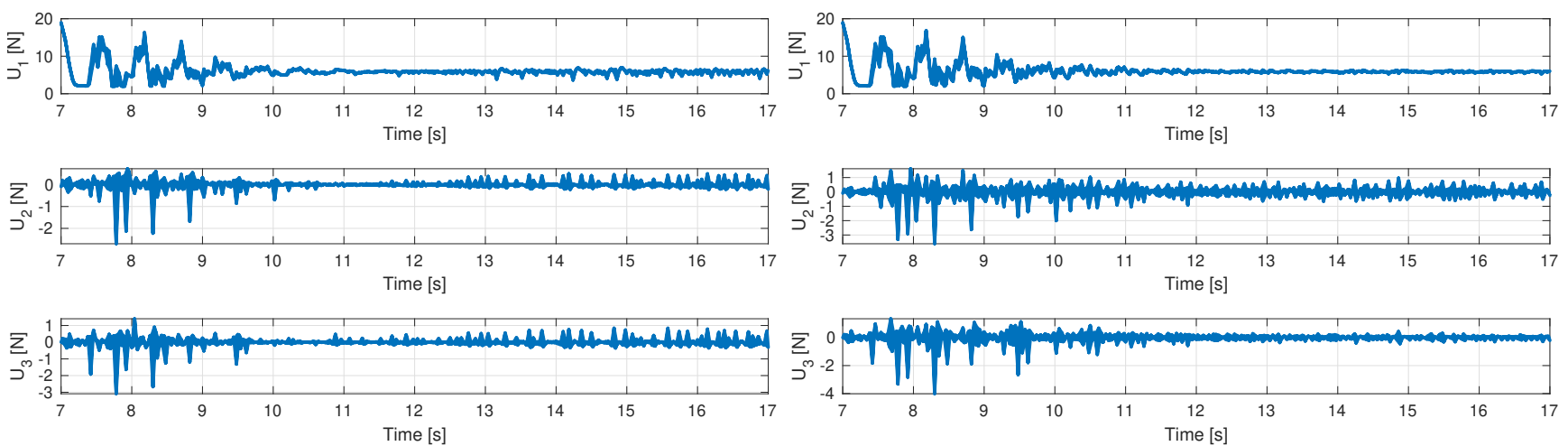

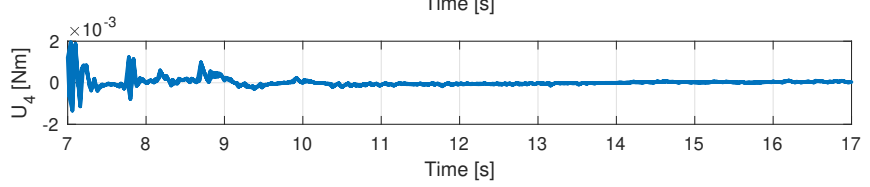

(a) VTOL inputs: bound on uncertainty $\bar{d}=3.8 \cdot 10^{-4}$

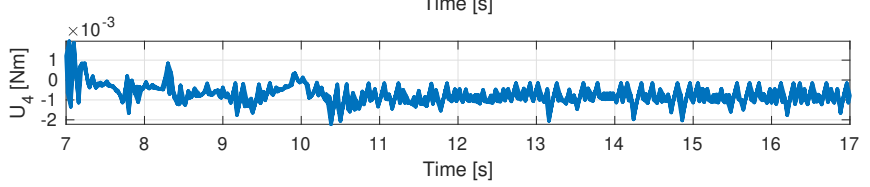

(b) VTOL inputs: bound on uncertainty $\tilde{d}=1.0 \cdot 10^{-1}$

Fig. 13: VTOL input signals in 2 different scenarios: on the left side the case when transmission delays and dropouts are considered and also disturbances are acting on the system, with bound on disturbances $\bar{d}=3.8 \cdot 10^{-4}$. On the right side the case when the bound on the disturbances is $\tilde{d}=1.0 \cdot 10^{-1}$ (the communication network has the same configuration as in the previous case).

- bounds on the maximum number of consecutive dropouts in the up-link and in the down-link, respectively: $\bar{L}_{s c}=3$, $\bar{L}_{c a}=4$

- bound on the data transmission delay between the two interfaces of the down-link: $\bar{\tau}_{s c}=5$

- bound on the data transmission delay between the two interfaces of the up-link: $\bar{\tau}_{c a}=4$

- control horizon $N=12$ and MTBS buffer length 21

- maximum additive uncertainty bound (according to Theorem 5.1 and taking into account only angular and translational acceleration components): $\bar{d}=3.8 \cdot 10^{-4}$.

The NMPC \& MBSF-PCS strategy is able to cope with (a priori) bounded network delays and packet dropouts. As expected, greater the maximum network delay or the maximum number of packet dropouts, poorer the control system performance, but the practical stability is still guaranteed.

Remark on the uncertainty bound: As noted in Remark 6.1, the bound $\bar{d}$ is conservative; we obtained a practical stable behavior for the VTOL applying the NMPC \& MBSF-PCS control strategy considering disturbances/model uncertainties greater, in absolute value, than the $\bar{d}$ value obtained applying the Theorem 5.1. For example, Fig. 12 describes the behavior of the VTOL system, controlled by mean of the proposed NMPC \& MBSF-PCS scheme with the same control and network configuration as in the experiment described above in Fig. 9, but considering $\tilde{d}=0.1$ as bound to the (random) disturbance acting on each angular and translational acceleration component of the VTOL model in Eq. (41). Note that, even if the controller is not able to impose to the VTOL to reach the desired equilibrium state, the VTOL state keeps moving quite close to the desired equilibrium condition, despite the disturbances acting on the system. Note also that (by comparing Fig. 11(a), 13(a) and 13(b)), due to the larger disturbance acting on the system in the second scenario, the control inputs $U_{2}, U_{3}$ and $U_{4}$ have an higher amplitude and are varying faster in the latter case (as expected).

\section{CONCLUding Remarks}

In this paper, the stabilization of a class of networked control systems consisting of a nonlinear dynamic system in which the loop is closed over a lossy communication network has been considered. One of the major issues when dealing with the stability of networked systems stems from the presence of asynchronous communications networks to implement the control law. In this respect, it is well known that a system like this is prone to instability when controlled by a feedback law designed without accounting for the timevarying delays and packet dropouts caused by the network, if no proper provisions are adopted. In this work, we have shown that by exploiting the packet transmission capabilities of the network it is possible to preserve the Regional-ISS property of the nominal controller by forwarding sequences of control moves. The possible loss of synchronization between the controller and the sensor/actuator nodes is addressed by a time-stamping policy, while the prediction consistency of the scheme is guaranteed by a move-blocking strategy which permits the controller to exactly know the true input sequence applied to the plant, even in absence of acknowledgments. Simulation results also comparing the proposed methodology with an existing one in the literature, show the effectiveness of the proposed networked nonlinear control scheme.

\section{REFERENCES}

[1] W. Zhang, M. Branicky, and S. Phillips, "Stability of networked control systems," IEEE Control Systems Magazine, vol. 21, no. 1, pp. 84-99, 2001.

[2] P. Antsaklis and J. Baillieul, "Guest editorial: Special issue on networked control systems," IEEE Transaction on Automatic Control, vol. 49, 2006. 
[3] J. Hespanha, P. Naghshtabrizi, and Y. Xu, "A survey of recent results in networked control systems," Proc. of IEEE Special Issue on Technology of Networked Control Systems, vol. 95, no. 1, pp. 138-162, 2007.

[4] W. Heemels, A. Teel, N. V. D. Wouw, and D. Nešić, "Networked control systems with communication constraints: Tradeoff between transmission intervals, delays and performance," IEEE Trans. Automatic Control, vol. 55, pp. 1781-1796, 2010.

[5] A. Bemporad, H.Heemels, and M.Johansson, Networked Control Systems. Springer-Verlag. New York., 2010.

[6] M. Törngren, "Fundamentals of implementing real-time control applications in distributed computer systems," J. of Real-Time Systems, vol. 14, pp. 219-250, 1998.

[7] J. Nilsson, B. Bernhardsson, and B. Wittenmark, "Stochastic analysis and control of real-time systems with random time delays," Automatica, vol. 34 , no. 1 , pp. $57-64,1998$.

[8] E. Lee, "Cyber physical systems: Design challenges," in Object Oriented Real-Time Distributed Computing (ISORC), 2008 11th IEEE International Symposium on, may 2008, pp. $363-369$.

[9] E. A. Lee, "Cyber-physical systems - are computing foundations adequate?" in Position Paper for NSF Workshop On Cyber-Physical Systems: Research Motivation, Techniques and Roadmap, October 2006.

[10] D. Georgiev and D. Tilbury, "Packet-based control: The H2-optimal solution," Automatica, vol. 42, pp. 127-144, 2006.

[11] G. Liu, J.Mu, D.Rees, and S.Chai, "Design and stability analysis of networked control systems with random communication time delay using the modified MPC," International Journal of Control, vol. 79, pp. 288297, 2006.

[12] G. Liu, Y.Xia, J.Che, D.Rees, and W.Hu, "Networked predictive control of systems with random network delays in both forward and feedback channels," IEEE Transactions on Industrial Electronics, vol. 54, pp. 1282-1297, 2007.

[13] L. Schenato, B. Sinopoli, M. Franceschetti, K. Poolla, and S. Sastry, "Foundations of control and estimation over lossy networks," Proceedings of the IEEE, vol. 95, no. 1, pp. 163-187, 2007.

[14] Y. Tipsuwan and M. Chow, "Control methodologies in networked control systems," Control Engineering Practice, vol. 11, pp. 1099-1111, 2003.

[15] A. Casavola, F.Mosca, and M.Papini, "Predictive teleoperation of constrained dynamic system via internet-like channels," in IEEE Trans Contr. Syst. Technol., 2006, pp. 681-694.

[16] E. Garone, B. Sinopoli, and A. Casavola, "LQG control over multichannel TCP-like erasure networks with probabilistic packet acknowledgements," in Proceedings of IEEE Conference on Decision and Control, Cancun, 2008.

[17] O. Imer, S. Yuksel, and T.Basar, "Optimal control of LTI systems over unreliable communication links," Automatica, vol. 42, pp. 1429-1439, 2006.

[18] M. Heemelsand, A. R. Teeland, N. van de Wouwand, and D. Nešić, "Networked control systems with communication constraints: Tradeoffs between transmission intervals, delays and performance," IEEE Transaction on Automatic Control, vol. 55, no. 8, pp. 1781-1796, 2010.

[19] L. Montestruque and P. Antsaklis, "On the model-based control of networked systems," Automatica, vol. 39, pp. 1837-1843, 2004.

[20] — "Stability of model-based networked control systems with timevarying transmission times," IEEE Trans. Automatic Control, vol. 49 , pp. 1562-1572, 2004

[21] D. Nešić and A. R. Teel, "Input-output stability properties of networked control systems," IEEE Trans. on Automatic Control, vol. 49, no. 10, pp. 1650-1667, 2004.

[22] G. Walsh and Y. Hong, "Scheduling of networked control systems," IEEE Control Systems Magazine, vol. 21, no. 1, pp. 57-65, 2001.

[23] D. Quevedo, E.Silva, and G.Goodwin, "Control over unreliable networks affected by packet erasures and variable transmission delays," IEEE Journal on Selected Areas in Communication, vol. 26, no. 4, pp. 672685, 2008.

[24] D. Quevedo and D. Nešić, "Input-to-state stability of packetized predictive control over unreliable networks affected by packet-dropouts," IEEE Trans. on Automatic Control, vol. 99, 2011.

[25] D. Quevedo and D. Nešić, "Robust stability of packetized predictive control of nonlinear systems with disturbances and markovian packet losses," Automatica, vol. 48, no. 8, 2012.

[26] G. Alldredge, M. Branicky, and V. Liberatore, "Play-back buffers in networked control systems: Evaluation and design," in Proc. American Control Conference, Seattle, 2008, pp. 3106-3113.

[27] P. Tang and C. de Silva, "Compensation for transmission delays in an ethernet-based control network using variable horizon predictive control," IEEE Trans. on Control Systems Technology, vol. 14, pp. 707716,2006
[28] I. Polushin, P. Liu, and C. Lung, "On the model-based approach to nonlinear networked control systems," in Proc. American Control Conference, 2007.

[29] L. Grüne, J.Pannek, and K. Worthmann, "A prediction based control scheme for networked systems with delays and packet dropouts," in Proc. 48th IEEE Conf. Decision and Control, Shanghai, China, 2009, pp. 537-542.

[30] A. Bemporad, "Predictive control of teleoperated constrained systems with unbounded communication delays," in Proc. IEEE Conf. on Decision and Control, 1998, pp. 2133-2138.

[31] J. Liu, D. Muñoz de la Peña, and P. Christofides, "Distributed model predictive control of nonlinear systems subject to asynchronous and delayed measurements," Automatica, vol. 46, no. 1, pp. 52-61, 2010.

[32] R. Findeisen and P. Varutti, "Stabilizing nonlinear predictive control over nondeterministic communication networks," In Nonlinear Model Predictive Control: Towards New Challenging Applications, vol. 384, pp. 167-179, Series: Lecture Notes in Control and Information Sciences. Magni, L.; Raimondo, D. M.; Allgöwer, F.(Eds.). Springer, 2009.

[33] G. Pin, M. Filippo, and T. Parisini, "Networked MPC for constrained linear systems: a recursive feasibility approach." in Proc. of the IEEE Conf. on Decision and Control, Shanghai, 2009, pp. 555 - 560.

[34] G. Pin and T. Parisini, "Networked predictive control of constrained nonlinear systems: Recursive feasibility and input-to-state stability analysis," in Proc. American Control Conference, 2009, pp. 2327 - 2334.

[35] - "Networked predictive control of uncertain constrained nonlinar systems: Recursive feasibility and input-to-state stability analysis," IEEE Trans. on Automatic Control, vol. 56, no. 1, pp. 72-87, 2011.

[36] R. Luck and A. Ray, "An observer-based compensator for distributed delays," Automatica, vol. 26, no. 5, pp. 903-908, 1990.

[37] H. Chan and Ü. Özgüner, "Closed-loop control of systems over a communication network with queues," Int. J. Control, vol. 62, no. 3, pp. 493-510, 1995

[38] I. Polushin, P. Liu, and C. Lung, "On the model-based approach to nonlinear networked control systems," Automatica, vol. 44, no. 9, pp. 2409-2414, 2008.

[39] A. Hekler, J. Fisher, and U. Hanebeck, "Sequence-based control for networked control systems based on virtual control inputs," Systems and Control Letters. To appear, 2012

[40] X. Zhang, X. Tang, and J. Chen, "Time synchronization of hierarchical real-time networked CNC system based on ethernet/internet," The International Journal of Advanced Manufacturing Technology, vol. 36, no. 11-12, pp. 1145-1156, 2008

[41] P. Varutti and R. Findeinsen, "On the synchronization problem for the stabilization of networked control systems over nondeterministic networks," in Proc. American Control Conference, St.Louis, MO, 2009, pp. 2216-2221.

[42] Z. P. Jiang and Y. Wang, "Input-to-state stability for discrete-time nonlinear systems," Automatica, vol. 37, no. 6, pp. 857-869, 2001.

[43] J. Baillieul and P. Antsaklis, "Control and communication challenges in networked real-time systems," Proceedings of the IEEE, vol. 95, pp. 9-28, 2007.

[44] B. Sundararaman, U. Buy, and A. Kshemkalyani, "Clock synchronization for wireless sensor networks: a survey," Ad Hoc Networks, vol. 3, no. 3, pp. 281-323, 2008

[45] S. Yoon and M. L. Sichitiu, "Tiny-sync: Tight time synchronization for wireless sensor networks," ACM Transactions on Sensor Networks, vol. 3, no. 2, 2007

[46] Z. Jiang, E.Sontag, and Y.Wang, "Input-to-state stability for discretetime nonlinear systems," in Proc. of the 14th IFAC World Congress, Beijing, 1999, pp. 277-282.

[47] J. Liu, D. Munoz de la Pena, P. D. Christofides, and J. F. Davis, "Lyapunov-based model predictive control of nonlinear systems subject to time-varying measurement delays," International Journal of Adaptive Control and Signal Processing, vol. 23, no. 8, pp. 788-807, 2009.

[48] H. Fogler, Elements of Chemical Reaction Engineering, 3rd ed. Prentice-Hall Inc., New Jersey, 1999.

[49] S. Bouabdallah, "Design and control of quadrotors with application to autonomous flying," Ph.D. dissertation, EPFL STI, Lausanne, 2007.

[50] S. Bouabdallah, P. Murrieri, and R. Siegwart, "Design and control of an indoor micro quadrotor," in Robotics and Automation, 2004. Proceedings. ICRA '04. 2004 IEEE International Conference on, vol. 5, April 2004, pp. 4393-4398 Vol.5.

[51] S. Bouabdallah and R. Siegwart, "Backstepping and sliding-mode techniques applied to an indoor micro quadrotor," in Proceedings of the 2005 IEEE International Conference on Robotics and Automation, April 2005. pp. 2247-2252. 
[52] — "Full control of a quadrotor," in 2007 IEEE/RSJ International Conference on Intelligent Robots and Systems, Oct 2007, pp. 153-158.

[53] T. Parisini, M. Sanguineti, and R. Zoppoli, "Nonlinear stabilization by receding horizon neural regulators," Int. J. of Control, vol. 70, no. 3, pp. 341-362, 1998.

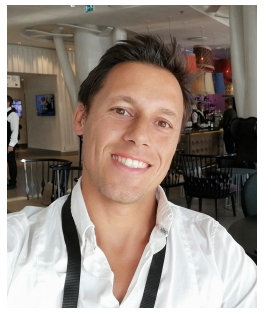

Gilberto Pin received the Laurea (M.Sc.) degree in Electrical Engineering (with honors) and the Ph.D. in Information Engineering from the University of Trieste, Italy, in 2005 and 2009, respectively. From 2009 to 2012 he was an Automation Engineer at Danieli Automation S.p.A., Italy. From 2012 to 2019, he has been a Energy Conversion Engineer at the Global Connectivity and Technology Center of Electrolux Italia S.p.A. Since 2019, he joined the Dept. of Information Engineering, University of Padova, Italy as Associate Professor. He is author of several papers published in international conferences and scientific journals concerning systems theory, signal processing methods and control applications. He is a co-recipient of the IFAC Best Application Paper Prize of the Journal of Process Control, Elsevier, for the three-year period 20112013. He serves as Associate Editor of the IEEE Trans. on Control Systems Technology and as a member of the IEEE CSS Conference Editorial Board. His current research interests include networked control, model predictive control, advanced system identification methods and deadbeat observers design. His activity is also devoted to the industrial application of advanced control techniques.

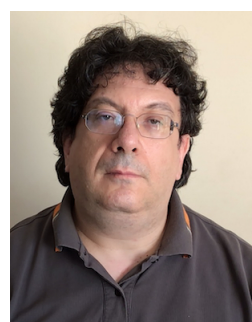

Gianfranco Fenu received the "Laurea" degree (M.Sc. degree) in Electronic Engineering and the $\mathrm{PhD}$. degree in Information Engineering from the University of Trieste, respectively in 1996 and 2001. From October 1997 to March 1998 he was visiting $\mathrm{PhD}$ student at the Fachgebiet Mess- und Regelungstechnik (Institute for Measurement and Automatic Control) of the University of Duisburg (Germany), led by Prof. Paul M. Frank. From November 1999 he is an Assistant Professor in the Engineering and Architecture of University of Trieste. His research interests include control theory, fault diagnosis, machine learning and robotics.

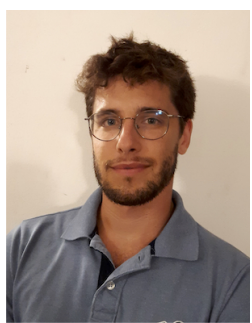

Vittorio Casagrande Vittorio Casagrande was born in Portogruaro (Italy) in 1992. He received the B.Sc. in Industrial Engineering in 2016 and the M.Sc. degree (cum laude) in Electrical Energy and System Engineering in 2019 both from University of Trieste (Italy). During his master, he studied for six months at Eindhoven University of Technology (TU/e) as an Erasmus student and he has been an intern at Danieli Automation S.p.A. from June 2018 to January 2019. After a brief experience as research associate at University of Trieste, he started a PhD at University College London (UCL) in September 2019. His main research interests are distributed optimization, model predictive control and machine learning.

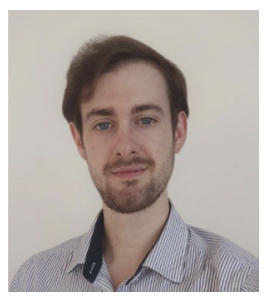

Davide Zorzenon Davide Zorzenon received the B.Sc. in industrial engineering and the M.Sc. degree (cum laude) in electrical energy and systems engineering from the University of Trieste, Italy, respectively in 2016 and 2019. Since July 2019, he is a doctoral candidate at the Control Systems Group at Technische Universität Berlin, Germany. His research interests include discrete event systems, maxplus algebra, model predictive control and machine learning.

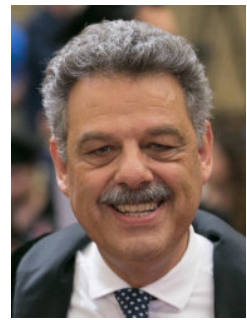

Thomas Parisini (F'11) received the Ph.D. degree in Electronic Engineering and Computer Science in 1993 from the University of Genoa. He was with Politecnico di Milano and since 2010 he holds the Chair of Industrial Control and is Director of Research at Imperial College London. He is a Deputy Director of the KIOS Research and Innovation Centre of Excellence, University of Cyprus. Since 2001 he is also Danieli Endowed Chair of Automation Engineering with University of Trieste. In 20092012 he was Deputy Rector of University of Trieste. In 2018 he received an Honorary Doctorate from University of Aalborg, Denmark. He authored or co-authored more than 320 research papers in archival journals, book chapters, and international conference proceedings. His research interests include neural-network approximations for optimal control problems, distributed methods for cyber-attack detection and cyber-secure control of large-scale systems, fault diagnosis for nonlinear and distributed systems, nonlinear model predictive control systems and nonlinear estimation. $\mathrm{He}$ is a co-recipient of the IFAC Best Application Paper Prize of the Journal of Process Control, Elsevier, for the three-year period 2011-2013 and of the 2004 Outstanding Paper Award of the IEEE Trans. on Neural Networks. He is also a recipient of the 2007 IEEE Distinguished Member Award. In 2016, he was awarded as Principal Investigator at Imperial of the H2020 European Union flagship Teaming Project KIOS Research and Innovation Centre of Excellence led by University of Cyprus. In 2012, he was awarded an ABB Research Grant dealing with energy-autonomous sensor networks for selfmonitoring industrial environments. Thomas Parisini currently serves as 2020 President-Elect of the IEEE Control Systems Society and has served as VicePresident for Publications Activities. During 2009-2016 he was the Editorin-Chief of the IEEE Trans. on Control Systems Technology. Since 2017, he is Editor for Control Applications of Automatica and since 2018 he is the Editor in Chief of the European Journal of Control. He is also the Chair of the IFAC Technical Committee on Fault Detection, Supervision \& Safety of Technical Processes - SAFEPROCESS. He was the Chair of the IEEE Control Systems Society Conference Editorial Board and a Distinguished Lecturer of the IEEE Control Systems Society. He was an elected member of the Board of Governors of the IEEE Control Systems Society and of the European Control Association (EUCA) and a member of the board of evaluators of the 7th Framework ICT Research Program of the European Union. Thomas Parisini is currently serving as an Associate Editor of the Int. J. of Control and served as Associate Editor of the IEEE Trans. on Automatic Control, of the IEEE Trans. on Neural Networks, of Automatica, and of the Int. J. of Robust and Nonlinear Control. Among other activities, he was the Program Chair of the 2008 IEEE Conference on Decision and Control and General Co-Chair of the 2013 IEEE Conference on Decision and Control. Prof. Parisini is a Fellow of the IEEE and of the IFAC. 